\title{
Characteristics of Supergene Gold of Karst Cavities of the Khokhoy Gold Ore Field (Aldan Shield, East Russia)
}

\author{
Galina S. Anisimova *D, Larisa A. Kondratieva and Veronika N. Kardashevskaia \\ Diamond and Precious Metal Geology Institute, SB RAS, 677000 Yakutsk, Russia; lkon12@yandex.ru (L.A.K.); \\ kardashevskaya92@mail.ru (V.N.K.) \\ * Correspondence: gsanisimova@diamond.ysn.ru; Tel.: +7-4112-33-58-72
}

Received: 20 December 2019; Accepted: 3 February 2020; Published: 6 February 2020

check for updates

\begin{abstract}
Typomorphic features of supergene gold in karst cavities were studied in the recently discovered $\mathrm{Au}-\mathrm{Te}-\mathrm{Sb}-\mathrm{Tl}$ deposit within the Khokhoy gold ore field of the Aldan-Stanovoy auriferous province (Aldan shield, East Russia). Two morphological types of supergene gold, massive and porous, are recognized there. The first type is represented by gold crystals and irregular mass, with the fineness ranging from 835 to $1000 \%$. They are closely associated with goethite, siderite, unnamed $\mathrm{Fe}$, Te, and $\mathrm{Tl}$ carbonates, $\mathrm{Tl}$ tellurites/tellurates and antimonates, as well as avicennite with a Te impurity. The second type is represented by mustard gold of two types with different internal structure: microporous and dendritic. The supergene gold is characterized by persistently high fineness. Along with $\mathrm{Ag}$, it invariably contains $\mathrm{Hg}$ (up to $5.78 \mathrm{wt} \%$ ) and $\mathrm{Bi}$, and, rarely, $\mathrm{Pb}, \mathrm{Cu}$, and Fe. The supergene gold is chemically homogeneous, and its particles are all marked by high fineness, without any rims or margins. The obtained characteristics made it possible to prove the existence of two genetic types of supergene gold. Mustard microporous gold is the result of the decomposition of the associated minerals—goethite, $\mathrm{Tl}$ oxides, tellurium, Fe, Mn and Tl carbonates and antimonates, containing microinclusions of gold. Massive gold and dendrites are newly formed. The decomposition, remobilization, and reprecipitation of residual gold nanoparticles and their aggregation led to the formation of dendrites, and with further crystal growth and filling of pores, to gold of massive morphology. In terms of morphology, internal structure, fineness, and trace element composition, supergene gold of the Khokhoy gold ore field is comparable to gold from the Kuranakh deposit (Russia) and the Carlin-type gold deposits. It also is similar to spungy and mustard gold from $\mathrm{Au}-\mathrm{Te}$ and $\mathrm{Au}-\mathrm{Sb}$ deposits, weathering crusts, and placers. Its main characteristic feature is a close paragenesis with $\mathrm{Tl}$ minerals.
\end{abstract}

Keywords: gold ore field; karst cavities; monolithic and porous gold; $\mathrm{Tl}$ oxides (avicennite); $\mathrm{Tl}$ carbonates; Tl tellurates and antimonates

\section{Introduction}

The importance of gold-bearing supergene zones increased significantly in relation to the discovery and commercial exploitation of the Carlin-type gold deposits (USA) [1-5]. In Russia, assigned to this type are deposits localized in karst cavities such as the Kuranakh deposit within the Central Aldan district of Yakutia and the Vorontsovskoe deposit in the Urals [6-9]. The question of native gold formation in supergene conditions, including in karst cavities, remains are as yet poorly investigated. Supergene gold in karst cavities has specific features, exhibits various textural and chemical characteristics that can help in elucidating the process of formation of supergenic gold.

That's why studies on the typomorphic features of the recently discovered supergene gold from the Khokhoy gold ore field within the Aldan-Stanovoy auriferous province are quite topical now. 


\section{Brief Geological Characteristics of the Khokhoy Ore Field}

The Khokhoy gold ore field is located in the Verkhneamginskaya auriferous zone, in the up-stream basin of the river Khokhoy, a right tributary of the river Amga [10]. The territory is a part of the Aldan-Stanovoy shield, in the basemen subsidence and sedimentary cover enlargement areas (Aldan shield, East Russia) (Figure 1). The majority of the sediments are those of Lower Cambrian terrigenous-carbonate sediments (predominantly dolomitic ones) with stratigraphic mismatches of closed Lower Jurassic terrigenous sandstone sediments. Mesozoic magmatism resulted in little stratified intrusions, monzonite lakkolithes and syenite-porphyries as well as in alkaline gabbroides. The ore field is structured by a vast faulted area with the North-Eastern strike. The faults' kinematics implies them being normal faults and slip faults with the amplitude of more than $100 \mathrm{~m}$ with echelon faults of the North-Western strike forming a ladder-shaped block structure of the ore field. Mineralization takes place where the echelon strippings intersect each other within the tectonized contact of Cambric and Jurassic sediments. Ores are accumulated in the karst cavities forming a vast zone with the North-Western strike of more than $10 \mathrm{~km}$ crossing the ore field from the South to the North. Almost the whole anomalous zone consists of karst holes of various size and mineralization extent, open as well as closed with sandstones. The latitude of the karst cavities opened during the mine working ranges from 5-15 to 50 more than $50 \mathrm{~m}$, the depth is $15-45 \mathrm{~m}$ or more. Karst cavities are formed of intensive limonitized argillaceous-sandy fulvous matter with primary ores and enclosing rocks fragments of various size. According to X-ray phase and thermal analyses, karst include quartz, potassium feldspars, muscovite, goethite, and less often calcite, hematite, and fluorite. Clinochlor, Fe-clinochlor, lepidocrocite, jarosite and kaolinite also attested. Primary ores exist as fragments of jasperoids, pyrite-adular-quartz metasomatites (Figure 2). The metasomatites contain $93.53-94.45 \%$ of $\mathrm{SiO}_{2}, 1.41-1.91 \%$ of $\mathrm{Al}_{2} \mathrm{O}_{3}$ and $0.88-1.56 \%$ of $\mathrm{K}_{2} \mathrm{O}$. The primary ores almost exclusively consist of fine-grain quartz with some adular in the form of little, frequently idiomorphic interpositions with late druse quartz lenses, often with chalcedony rim.

The geochemical association of the Khokhoy gold ore field elements is the following $\mathrm{Au}, \mathrm{Sb}, \mathrm{Te}$ and Tl. The main minerals of karst cavities are of fine grain quartz, chalcedony, opal, adular, sericite, calcite, barite, fluorite, goethite, limonite, hematite. Unnamed $\mathrm{Fe}, \mathrm{Mn}, \mathrm{Te}$, and $\mathrm{Tl}$ carbonates, galena, weissbergite, berthierite, arsenopyrite, chalcocite, unnamed sulfide Re and $\mathrm{W}$, avicennite, hollandite, chalcopyrite, acanthite, chlorargyrite, fine grain native gold and silver are rarely. Gold mineralization of the Khokhoy gold ore field has a hypogene-supergene nature. The loose gold-bearing rocks here are secondary formations, which originated from oxidation, disintegration, and redeposition in the karst cavities of primary ores such as pyrite-adular-quartz metasomatites that resulted from silicic-potassic metasomatism of carbonate rocks. Strong $\mathrm{Tl}$ enrichment occurs during potassic metasomatism, in the fault zones, as is well seen in the Khokhoy gold ore field. The Au-Tl-As-Sb-Te-Ba geochemical profile of the mineralization and its low-temperature formative conditions suggest the epithermal origin of the ores. With regard to structural-morphological and mineralogical-geochemical parameters, gold mineralization of the Khokhoy gold ore field is comparable to the Kuranakh-type gold deposits of the Central Aldan district of Yakutia, representing unique supergene, shallow-depth, friable ores with free gold localized in karst cavities. They are characterized by large reserves with a relatively low gold grade. 


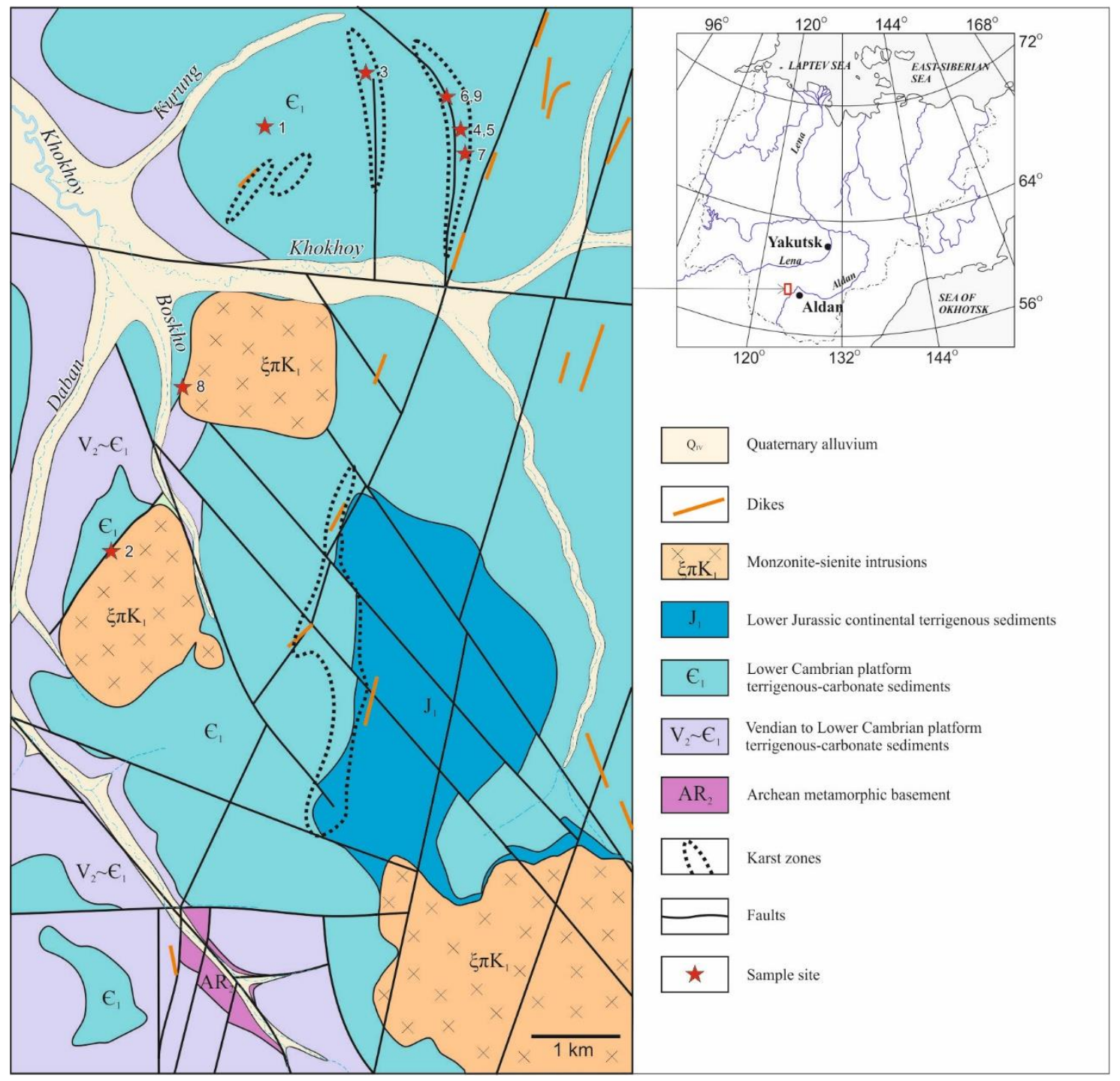

Figure 1. Structure of the Khokhoy gold ore field (Aldan shield, East Russia). 

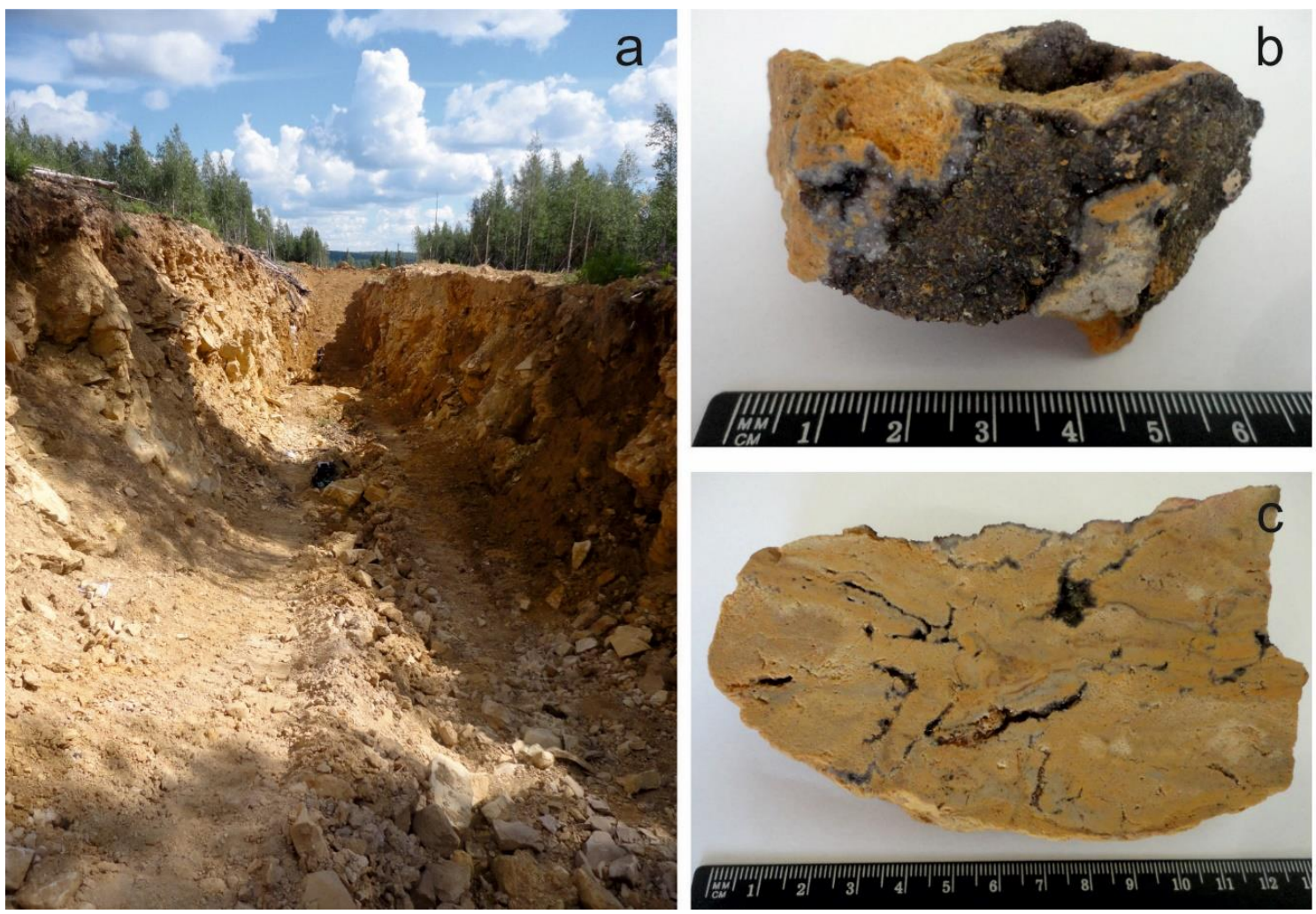

Figure 2. Ore bearing karst, (a) general look, (b) pyrite-adular-quartz jasperoids, (c) section of b.

\section{Materials and Methods}

We used grab samples and friable materials of argillaceous and sandy fragments, taken from the surface mines and the core drilling holes of the Khokhoy gold ore field karst (Table 1). The grab samples of 0.3-0.5 kg taken from the karst fragments were used for making polished slides. The friable materials of $3 \mathrm{~kg}$, on the other hand, were hydroseparated and to get the heavy residue. All samples were investigated with the use of binocular microscope, then we detected individual gold lumps and the associated minerals, which we forced together under pressure, made solid with epoxide and polished. All the polished samples were investigated with the use of Jenavert microscope in the reflected light, photographed and made ready for microprobe analysis. In order to investigate the chemical composition of the native gold and the associating minerals (ore, vein, and supergene minerals) as well as to detect unknown minerals we used microprobe analysis. The samples were analyzed with Camebax microanalyzer (Cameca, Courbevoie, France). We investigated the composition of gold making 3-5 probes in the center and on the edges of the gold lumps (the analysis was performed by N. Khristoforova). The majority of the samples were analyzed with the use of scanning electron microscope JEOL JSM-6480LV and energy spectrometer by Oxford (JEOL, Tokyo, Japan), which was used for taking the picture (the analysis was performed by S. Popova and S. Karpova). We made the quantitative XPP analyses using OXFORD INCA ENERGY 350 (Oxford Instruments, UK). The analysis conditions are as follows, the accelerating voltage of $20 \mathrm{kV}$, the measuring current flow is $1.08 \mathrm{nA}$, the measurement time is $10 \mathrm{~s}$. The photographing conditions are as follows, voltage is $20 \mathrm{kV}$, the current flow is $17 \mathrm{nA}$. The analytic lines are $\mathrm{Cu}, \mathrm{Fe}, \mathrm{Zn}-\mathrm{K} \alpha ; \mathrm{Sb}, \mathrm{S}-\mathrm{L} \alpha$. The standards are gold $750 \%$ - $\mathrm{Au}, \mathrm{Ag} ; \mathrm{HgTe}$ (coloradoite)—Hg, Te; $\mathrm{CuSbS}_{2}$ (chalcostibite)—Cu, Sb, S; ZnS (sphalerite)—Zn; $\mathrm{CuFeS}_{2}$ (chalcopyrite)—Fe; PbS (galena)—Pb; FeAsS (arsenopyrite)—As; $\mathrm{BaSO}_{4}$ (barite)—Ba; $\mathrm{ZrSiO}_{4}$ (zircon)-Zr; manganese $100 \%-\mathrm{Mn}$. 
Table 1. Details of samples studied.

\begin{tabular}{|c|c|c|c|c|c|c|c|}
\hline \multirow{2}{*}{ No. } & \multirow{2}{*}{ Sample Label } & \multicolumn{2}{|c|}{ Grid } & \multirow{2}{*}{ No. Grains } & \multirow{2}{*}{ Primary Gold } & \multicolumn{2}{|c|}{ Secondary Gold } \\
\hline & & Easting & Northing & & & Massive & Porous \\
\hline 1 & 4004 & 6555710 & 21514040 & 2 & 2 & 0 & 0 \\
\hline 2 & 3237 & 6550789 & 21510138 & 1 & 1 & 0 & 0 \\
\hline 3 & K-21 & 6556300 & 21513025 & 1 & 1 & 0 & 0 \\
\hline 4 & 12014 & 6556295 & 21501333 & 1 & 2 & 0 & 0 \\
\hline 5 & $\begin{array}{c}5-15-53,54,57-60,64 \\
65,67-74,76-78,80-82, \\
84-87,90,94-95,97-98, \\
100-103,105-108, \\
110-113,115-118,6-15\end{array}$ & 6555705 & 21514030 & 82 & 0 & 72 & 10 \\
\hline 6 & $1-17-1-7$ & 6556046 & 21513843 & 7 & 0 & 0 & 7 \\
\hline 7 & 1-17-8-14 & 6555603 & 21514221 & 7 & 0 & 0 & 7 \\
\hline 8 & $1-17-15$ & 6552872 & 21511211 & 1 & 0 & 0 & 1 \\
\hline 9 & $1-17-16-21$ & 6556022 & 21513960 & 6 & 0 & 0 & 6 \\
\hline
\end{tabular}

Limits of element detection (wt\%) X-ray spectral microprobe analysis: Au 0.117, Ag 0.061, $\mathrm{Hg}$ 0.083, $\mathrm{Cu}$ 0.031, Fe 0.019, $\mathrm{Pb}$ 0.066, Bi 0.095. Limits of element detection (wt\%) scanning electron microscope equipped with energy spectrometer: $\mathrm{Au} 1.84, \mathrm{Ag} \mathrm{0.96,} \mathrm{Hg} 1.6, \mathrm{Cu} 1.22, \mathrm{Fe} 1.04, \mathrm{~Pb} 1.78$, Bi 2.7.

\section{Results}

\subsection{Supergene Gold of the Khokhoy Gold Ore Field}

\section{Primary Native Gold}

Visually native gold in primary ores is extremely rare [10]. It is assumed that there is invisible finely divided gold, as evidenced by the gold content in the ore. Native gold is largely attested in porous oxidized pyrite relics or in quartz associated with barite and galena as submicroscopic cloddy effusions of the size up to $5 \mu \mathrm{m}$, which is why we could not make conditional analysis. The native gold on the whole barely contains any impurities (970-999\%o), which is consistent with the ratio of $\mathrm{Au} / \mathrm{Ag}$ in ores 100/1. Very rarely, grains with up to $15 \mathrm{wt} \%$ of silver are attested.

\subsection{Supergene Gold of the Karst Cavities}

The physicochemical processes that have taken place during the development of the karst have influenced the typomorphic characteristics of gold. The karst cavities gold is most frequently attested in loose condition. At the same time, it is bigger in its size than that of primary ores. The Khokhoy gold ore field gold is represented by two types: massive and porous.

\subsubsection{Massive Gold}

Massive gold is represented by crystals and irregular mass (Figures 3 and 4). Crystals are rarely attested in karst holes and make $1-2 \%$ of the total mass. They are represented by individuals of dodecahedral (Figure 3a) and octahedral (Figure 3b) shapes with clear cut facets, and rarely with smooth edges. On the surface of the facets some dints and dents indicating sliding processes are attested. The size of the crystals and irregular mass of gold is reaches $0.2 \mathrm{~mm}$. The fineness of the gold is very high, namely $950-1000 \%$ (Table 2; Nos. 1 and 2). 

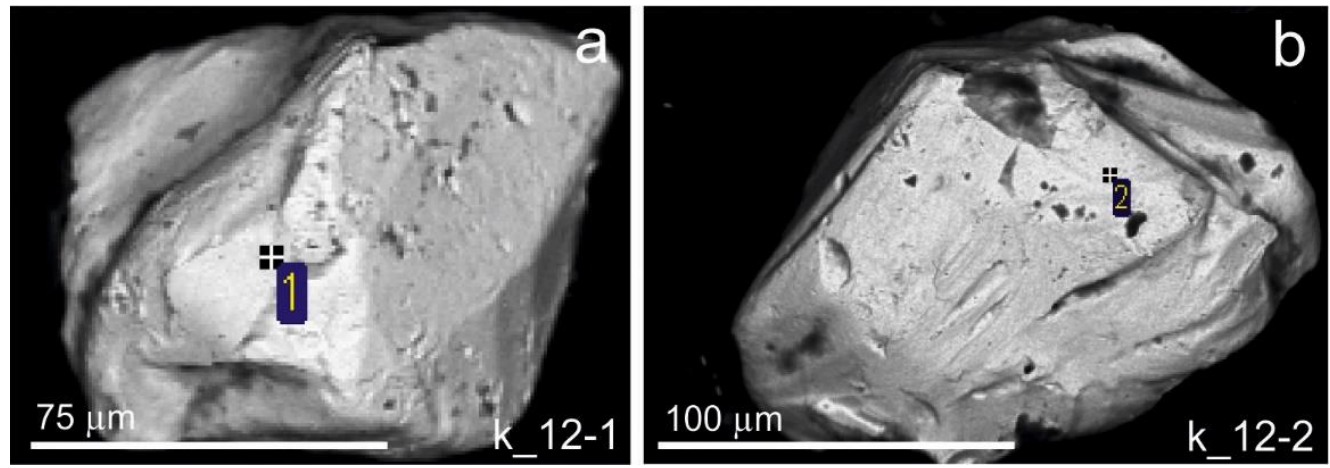

Figure 3. The dodecahedral (a) and octahedral (b) crystals of Khokhoy ore field. Mounted polished sections, scanning electronic microscope in back-scattering electron mode. The numbers in microphotographs are analitical spots, here and in other figures.
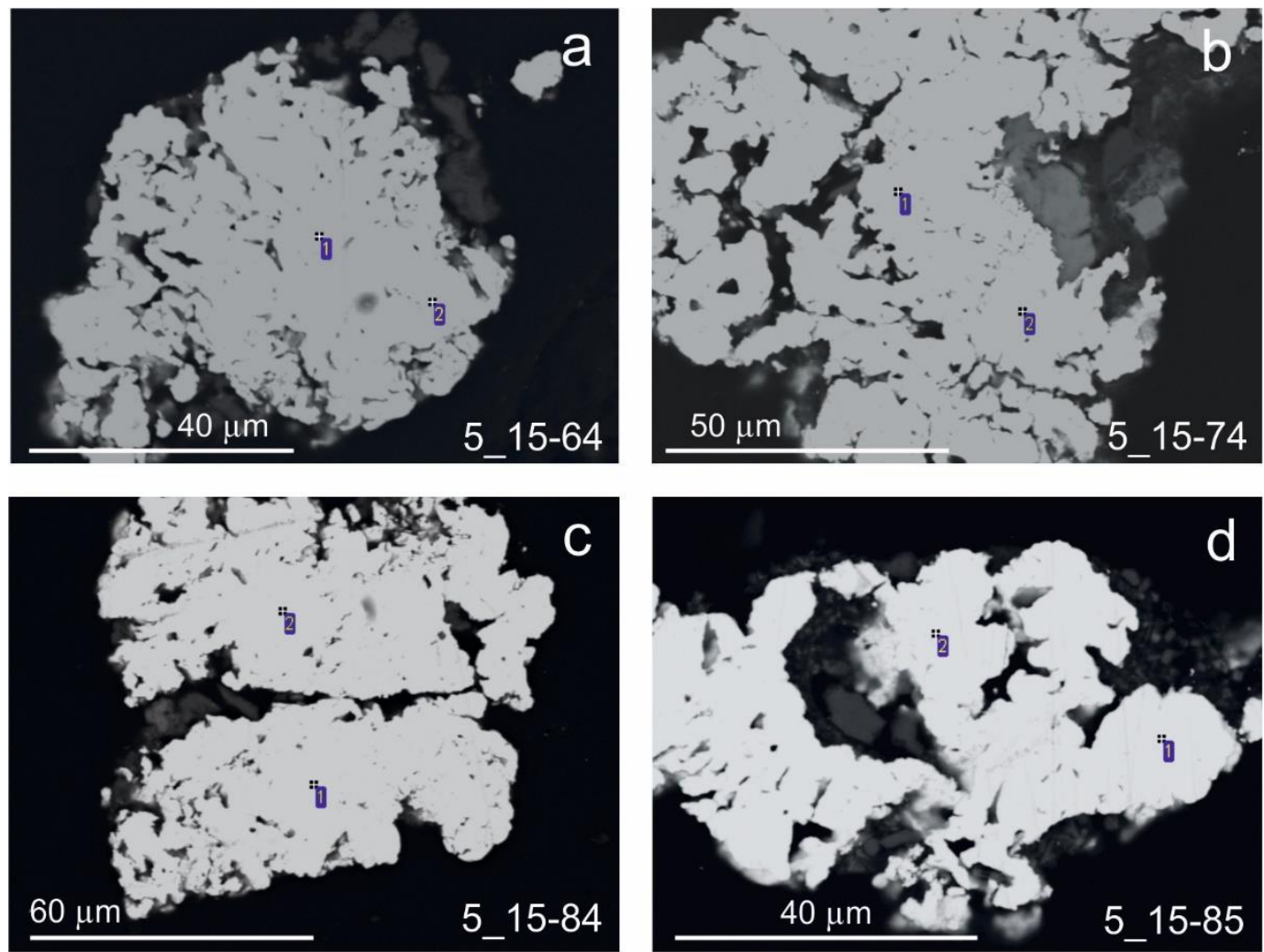

Figure 4. Massive gold. (a) gold with $\mathrm{Hg}$ alloy, (b,c) pure gold, (d) gold with Ag alloy. Mounted polished sections, scanning electronic microscope in back-scattering electron mode. 
Table 2. The composition (in wt $\%$ ) and fineness (in \%o) of the supergene gold shown in Figures 3, 4, 8 and 10 .

\begin{tabular}{|c|c|c|c|c|c|c|c|}
\hline No. & Sample * & Sp. & Ag & $\mathrm{Au}$ & $\mathbf{H g}$ & Total & Fineness \\
\hline 1 & \multirow{2}{*}{ K_12 } & 1 & - & 98.31 & - & 98.31 & 1000 \\
\hline 2 & & 2 & 4.73 & 94.28 & - & 99.01 & 952 \\
\hline 3 & \multirow{2}{*}{ 5_15-64 } & 1 & - & 94.01 & 3.61 & 97.62 & 963 \\
\hline 4 & & 2 & - & 95.90 & 2.92 & 98.82 & 970 \\
\hline 5 & \multirow{2}{*}{ 5_15-74 } & 1 & - & 100.48 & - & 100.48 & 1000 \\
\hline 6 & & 2 & 0.64 & 99.11 & - & 99.75 & 993 \\
\hline 7 & \multirow{2}{*}{ 5_15-84 } & 1 & - & 98.16 & - & 98.16 & 1000 \\
\hline 8 & & 2 & - & 98.39 & - & 98.39 & 1000 \\
\hline 9 & \multirow{2}{*}{ 5_15-85 } & 1 & 4.42 & 95.13 & - & 99.55 & 956 \\
\hline 10 & & 2 & - & 101.40 & - & 101.40 & 1000 \\
\hline 11 & \multirow{4}{*}{ 1_17-5 } & 1 & - & 101.78 & - & 101.78 & 1000 \\
\hline 12 & & 2 & - & 98.71 & - & 98.71 & 1000 \\
\hline 13 & & 3 & - & 99.08 & - & 99.08 & 1000 \\
\hline 14 & & 4 & - & 99.35 & - & 99.35 & 1000 \\
\hline 15 & \multirow{9}{*}{ 1_17-15 } & 1 & - & 95.56 & 4.57 & 100.13 & 954 \\
\hline 16 & & 3 & 0.28 & 96.64 & 3.54 & 100.47 & 962 \\
\hline 17 & & 4 & - & 100.29 & - & 100.29 & 1000 \\
\hline 18 & & 5 & - & 100.91 & - & 100.91 & 1000 \\
\hline 19 & & 6 & 0.37 & 96.37 & 2.85 & 99.59 & 967 \\
\hline 20 & & 7 & - & 95.50 & 4.74 & 100.24 & 953 \\
\hline 21 & & 8 & - & 96.75 & 3.08 & 99.83 & 969 \\
\hline 22 & & 9 & - & 98.85 & 1.56 & 100.41 & 984 \\
\hline 23 & & 10 & - & 93.91 & 4.33 & 98.24 & 956 \\
\hline 24 & \multirow{7}{*}{ 1_17-13 } & 1 & - & 98.45 & - & 98.45 & 1000 \\
\hline 25 & & 4 & - & 100.57 & - & 100.57 & 1000 \\
\hline 26 & & 5 & - & 99.77 & - & 99.77 & 1000 \\
\hline 27 & & 6 & - & 97.90 & - & 97.90 & 1000 \\
\hline 28 & & 7 & - & 97.25 & - & 97.25 & 1000 \\
\hline 29 & & 8 & - & 101.06 & - & 101.06 & 1000 \\
\hline 30 & & 9 & - & 99.11 & - & 99.11 & 1000 \\
\hline 31 & \multirow{6}{*}{ 1_17-18 } & 1 & - & 99.01 & - & 99.01 & 1000 \\
\hline 32 & & 2 & 5.98 & 92.21 & - & 98.19 & 939 \\
\hline 33 & & 3 & - & 100.12 & - & 100.12 & 1000 \\
\hline 34 & & 4 & 2.77 & 95.59 & - & 98.36 & 972 \\
\hline 35 & & 5 & - & 97.49 & - & 97.49 & 1000 \\
\hline 36 & & 6 & 6.08 & 92.56 & - & 98.64 & 938 \\
\hline 37 & \multirow{4}{*}{ 1_17-7 } & 2 & 0.59 & 95.41 & 2.57 & 98.57 & 968 \\
\hline 38 & & 6 & 1.25 & 98.76 & 0.61 & 100.62 & 982 \\
\hline 39 & & 8 & 0.95 & 97.18 & 2.68 & 100.81 & 964 \\
\hline 40 & & 9 & 2.03 & 95.51 & 3.04 & 100.58 & 949 \\
\hline 41 & \multirow{7}{*}{ 1_17-14 } & 1 & 1.97 & 97.67 & 1.54 & 100.18 & 975 \\
\hline 42 & & 2 & - & 100.08 & - & 100.08 & 1000 \\
\hline 43 & & 3 & - & 99.78 & - & 99.78 & 1000 \\
\hline 44 & & 4 & 3.14 & 92.32 & 3.01 & 98.48 & 937 \\
\hline 45 & & 5 & & 100.20 & - & 100.20 & 1000 \\
\hline 46 & & 7 & 2.95 & 95.42 & 1.17 & 99.54 & 958 \\
\hline 47 & & 8 & - & 95.05 & 4.33 & 99.38 & 956 \\
\hline 48 & \multirow{5}{*}{ 1_17-20 } & 2 & 5.40 & 93.43 & - & 98.83 & 945 \\
\hline 49 & & 4 & 3.99 & 97.98 & - & 101.97 & 961 \\
\hline 50 & & 5 & 3.13 & 96.10 & - & 99.24 & 968 \\
\hline 51 & & 6 & 2.18 & 98.61 & - & 100.78 & 978 \\
\hline 52 & & 7 & 2.54 & 96.86 & - & 99.40 & 974 \\
\hline 53 & \multirow{4}{*}{ 1_17-6 } & 3 & - & 98.02 & - & 98.02 & 1000 \\
\hline 54 & & 6 & - & 98.74 & - & 98.74 & 1000 \\
\hline 55 & & 7 & - & 98.78 & - & 98.78 & 1000 \\
\hline 56 & & 8 & - & 99.82 & - & 99.82 & 1000 \\
\hline
\end{tabular}

Notes: * Nos. 1-10—massive gold; 1-2—crystals; 3-10-irregular mass gold; 11-55—porous gold. Sp.—analysis spot in figures. The analyzes were performed on a scanning electron microscope equipped with energy spectrometer. 
The most widespread are cloddy gold particles of massive structure (Figure 4). In terms of morphology, they belong to the irregularly-shaped type, and associate closely with various minerals formed from the weathering of primary ores (Figure 5). The supergene gold often occurs in association with unnamed $\mathrm{Fe}, \mathrm{Te}$, and $\mathrm{Tl}$ carbonates (Figure 5a, Table 3; Nos. 1-8). Aggregates of intimately intergrown unnamed tellurates of $\mathrm{Tl}$ and native gold are occasionally observed along cracks in the gold particles (Figure 5b, Table 3; Nos. 9-15). In the interstices between massive gold there are found dendritic gold particles closely interwoven with goethite (Figure 5c, Table 3, Nos. 16-23). The most intricate forms of gold are seen in a very rare mineral avicennite $\left(\mathrm{Tl}_{2} \mathrm{O}_{3}\right)$, an oxide of thallium with a Te impurity (Figure $5 \mathrm{~d}$, Table 3; Nos. 24-39). Sometimes massive gold occurs in assemblage with siderite (Figure 5e,f, Table 3; Nos. 40-43) and goethite (Figure 6, Table 3, Nos. 14-15). At a higher magnification, micron gold inclusions are visible in associating minerals (Figure 5f, Table 3, Nos. 44-55; Figure 6, Nos. 9-13). At a higher magnification, micron-sized gold grains are seen in siderite (Figure 5f, Table 3; Nos. 44-55). Fineness of the monolithic gold varies from 835 to $1000 \%$, with the high-fineness particles prevailing (Tables 2 and 3). Along with Ag, trace elements include mercury (up to $3.61 \mathrm{wt} \%$ ), bismuth, and, more rarely $\mathrm{Fe}, \mathrm{Cu}, \mathrm{Zn}$, and $\mathrm{Pb}$ (Table 4).
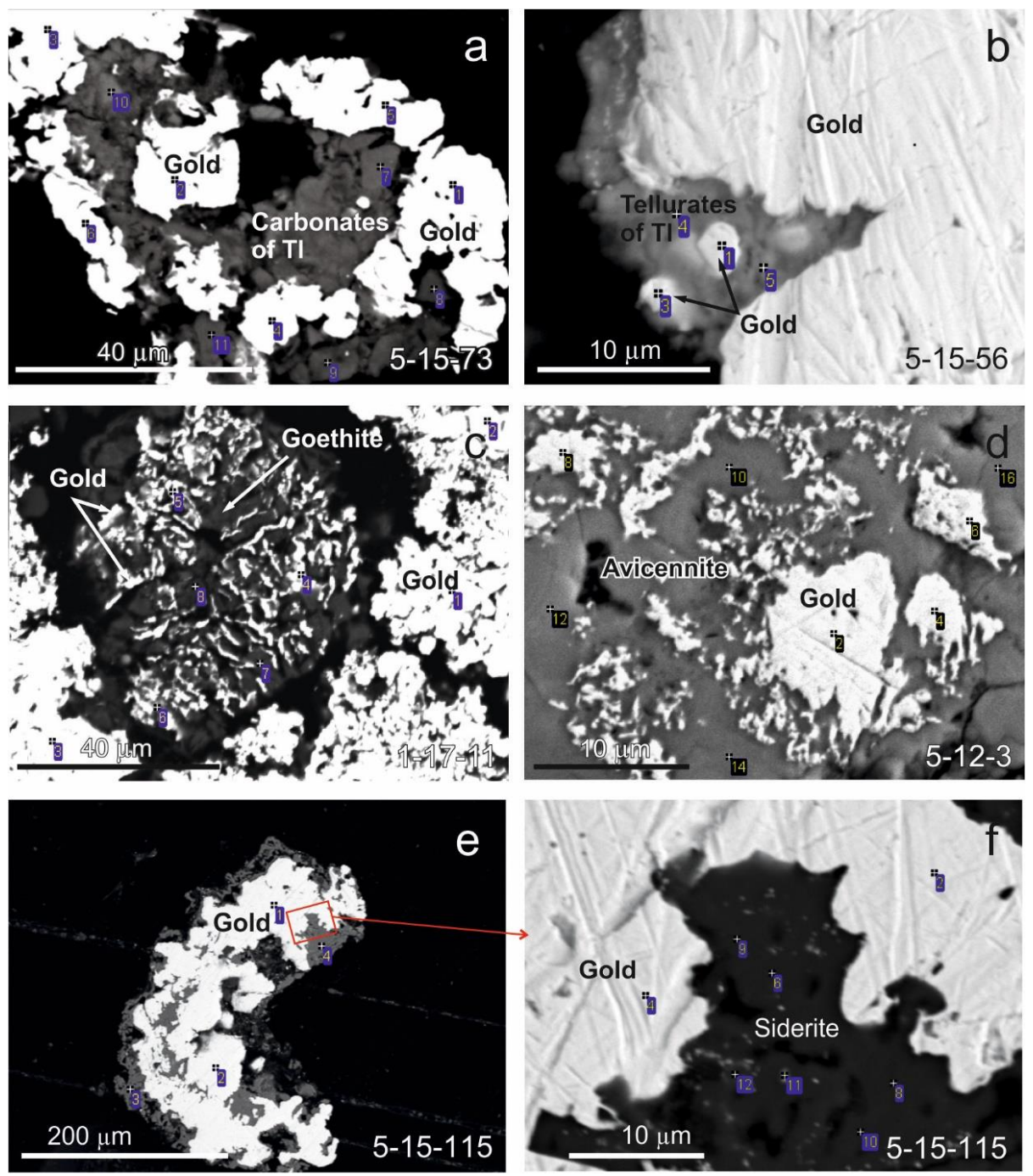

Figure 5. Massive gold in association with various supergene minerals. (a) massive gold in association with unnamed carbonates $\mathrm{Te}, \mathrm{Fe}, \mathrm{Tl}$, (b) massive gold with inclusions of unnamed tellurates $\mathrm{Tl}$, (c) honeycomb gold at the edges, sponge gold in the center in close association with goethite, (d) inclusion of massive and sponge gold in avicennite, (e) massive gold in association with siderite, (f) enlarged fragment of e. Mounted sections, scanning electron microscope, image in the back-scattering electron mode. 
Table 3. The composition of the massive gold and associated minerals (in wt\%) shown in Figures 5 and 6 .

\begin{tabular}{|c|c|c|c|c|c|c|c|c|c|c|c|}
\hline No. & Sample & Sp. & $\mathrm{Au}$ & Ag & $\mathrm{Hg}$ & $\mathrm{Te}$ & $\mathrm{Fe}$ & $\mathrm{Tl}$ & Si & $\mathrm{O}$ & Total \\
\hline 1 & 5_15_73 & 1 & 99.61 & - & 1.48 & - & - & - & - & - & 101.09 \\
\hline 2 & 5_15_73 & 2 & 101.61 & - & - & - & - & - & - & - & 101.61 \\
\hline 3 & 5_15_73 & 6 & 96.87 & 1.38 & 2.6 & - & - & - & - & - & 100.85 \\
\hline 4 & 5_15_73 & 7 & - & - & - & 13.73 & 11.23 & 22.78 & 1.53 & 17.78 & 67.05 \\
\hline 5 & 5_15_73 & 8 & - & - & - & 10.97 & 12.29 & 15.58 & 0.92 & 15.1 & 54.86 \\
\hline 6 & 5_15_73 & 9 & - & - & - & 13.13 & 12.44 & 20.99 & 2.51 & 17.26 & 66.33 \\
\hline 7 & 5_15_73 & 10 & - & - & - & 16.08 & 14.58 & 24.42 & & 16.08 & 71.16 \\
\hline 8 & 5_15_73 & 11 & - & - & - & 14.93 & 11.82 & 20.9 & 1.39 & 14.51 & 63.55 \\
\hline 9 & 5_15_101 & 1 & 50.27 & 3.62 & - & - & 23.15 & - & - & 23.06 & 100.1 \\
\hline 10 & 5_15_101 & 2 & 50.45 & 2.56 & - & - & 20.81 & - & - & 25.94 & 99.76 \\
\hline 11 & 5_15_101 & 3 & 52.45 & 2.6 & - & - & 20.57 & - & - & 23.26 & 98.88 \\
\hline 12 & 5_15_101 & 4 & 53.02 & 3.95 & - & - & 21.93 & - & - & 19.83 & 98.73 \\
\hline 13 & 5_15_101 & 5 & 51.43 & 1.64 & - & - & 24.96 & - & - & 30.32 & 108.35 \\
\hline 14 & 5_15_101 & 6 & 92.07 & 7.39 & - & - & - & - & - & - & 99.46 \\
\hline 15 & 5_15_101 & 7 & 93.01 & 6.74 & - & - & - & - & - & - & 99.75 \\
\hline 16 & 1_17_11 & 1 & 93.41 & 5.64 & - & - & - & - & - & - & 99.05 \\
\hline 17 & 1_17_11 & 2 & 98.17 & - & - & - & - & - & - & - & 98.17 \\
\hline 18 & 1_17_11 & 3 & 94.18 & 6.00 & - & - & - & - & - & - & 100.18 \\
\hline 19 & 1_17_11 & 4 & 93.28 & - & - & - & 1.68 & - & - & 4.01 & 98.97 \\
\hline 20 & 1_17_11 & 5 & 98.79 & - & - & - & - & - & - & - & 98.79 \\
\hline 21 & 1_17_11 & 6 & 97.45 & 2.33 & - & - & - & - & - & - & 99.78 \\
\hline 22 & 1_17_11 & 7 & - & - & - & 4.67 & 60.42 & - & - & 35.45 & 100.54 \\
\hline 23 & 1_17_11 & 8 & - & - & - & 5.48 & 58.33 & - & - & 35.23 & 99.04 \\
\hline 24 & 5_15_3 & 1 & 99.39 & - & - & - & - & - & - & - & 99.39 \\
\hline 25 & 5_15_3 & 2 & 99.39 & - & - & - & - & - & - & - & 99.39 \\
\hline 26 & 5_15_3 & 3 & 94.79 & 6.28 & - & - & - & - & - & - & 101.07 \\
\hline 27 & 5_15_3 & 4 & 93.91 & 6.42 & - & - & - & - & - & - & 100.33 \\
\hline 28 & 5_15_3 & 5 & 93.39 & 4.44 & - & - & - & - & - & - & 97.83 \\
\hline 29 & 5_15_3 & 6 & 91.78 & 5.77 & - & - & - & - & - & - & 97.55 \\
\hline 30 & 5_15_3 & 7 & 93.87 & 5.91 & - & - & - & - & - & - & 99.78 \\
\hline 31 & 5_15_3 & 8 & 93.13 & 4.56 & - & - & - & - & - & - & 97.69 \\
\hline 32 & 5_15_3 & 9 & - & - & - & 4.93 & - & 75.65 & - & 17.25 & 97.83 \\
\hline 33 & 5_15_3 & 10 & - & - & - & 3.96 & - & 81.6 & - & 13.65 & 99.21 \\
\hline 34 & 5_15_3 & 11 & - & - & - & 7.5 & - & 79.55 & - & 13.49 & 100.54 \\
\hline 35 & 5_15_3 & 12 & - & - & - & 5.15 & - & 83.25 & - & 13.37 & 101.77 \\
\hline 36 & 5_15_3 & 13 & - & - & - & 5.53 & - & 78.79 & - & 13.41 & 97.73 \\
\hline 37 & 5_15_3 & 14 & - & - & - & 3.85 & - & 81.09 & - & 13.04 & 97.98 \\
\hline 38 & 5_15_3 & 15 & - & - & - & 7.74 & - & 76.21 & - & 15.31 & 99.26 \\
\hline 39 & 5_15_3 & 16 & - & - & - & 4.87 & - & 81.91 & - & 16.16 & 102.94 \\
\hline 40 & 5_15-115 & 1 & 93.99 & 5.07 & - & - & - & - & - & - & 99.06 \\
\hline 41 & 5_15-115 & 2 & 92.51 & 6.95 & - & - & - & - & - & - & 99.46 \\
\hline 42 & 5_15-115 & 3 & - & - & - & - & 45.22 & - & 3.20 & 30.85 & 79.27 \\
\hline 43 & 5_15-115 & 4 & - & - & - & - & 45.28 & - & 2.23 & 30.24 & 77.75 \\
\hline 44 & 5_15-115f & 1 & 90.63 & 7.02 & - & - & - & - & - & - & 97.65 \\
\hline 45 & 5_15-115f & 2 & 91.13 & 6.80 & - & - & - & - & - & - & 97.93 \\
\hline 46 & 5_15-115f & 3 & 92.50 & 6.65 & - & - & - & - & - & - & 99.15 \\
\hline 47 & 5_15-115f & 4 & 90.57 & 7.56 & - & - & - & - & - & - & 98.13 \\
\hline 48 & 5_15-115f & 5 & - & - & - & - & 48.28 & - & 2.24 & 28.48 & 79.00 \\
\hline 49 & 5_15-115f & 6 & - & - & - & - & 47.20 & - & 2.10 & 27.50 & 76.80 \\
\hline 50 & 5_15-115f & 7 & - & - & - & - & 46.93 & - & 2.27 & 30.94 & 80.58 \\
\hline 51 & 5_15-115f & 8 & - & - & - & - & 48.23 & - & 2.32 & 30.48 & 81.03 \\
\hline 52 & 5_15-115f & 9 & - & - & - & - & 47.95 & - & 2.42 & 28.68 & 79.06 \\
\hline 53 & 5_15-115f & 10 & - & - & - & - & 48.55 & - & 2.07 & 30.76 & 81.38 \\
\hline 54 & 5_15-115f & 11 & 15.33 & 2.87 & - & - & 38.46 & - & 1.89 & 37.28 & 95.84 \\
\hline 55 & 5_15-115f & 12 & 15.66 & - & - & - & 41.85 & - & 2.23 & 36.90 & 96.64 \\
\hline
\end{tabular}



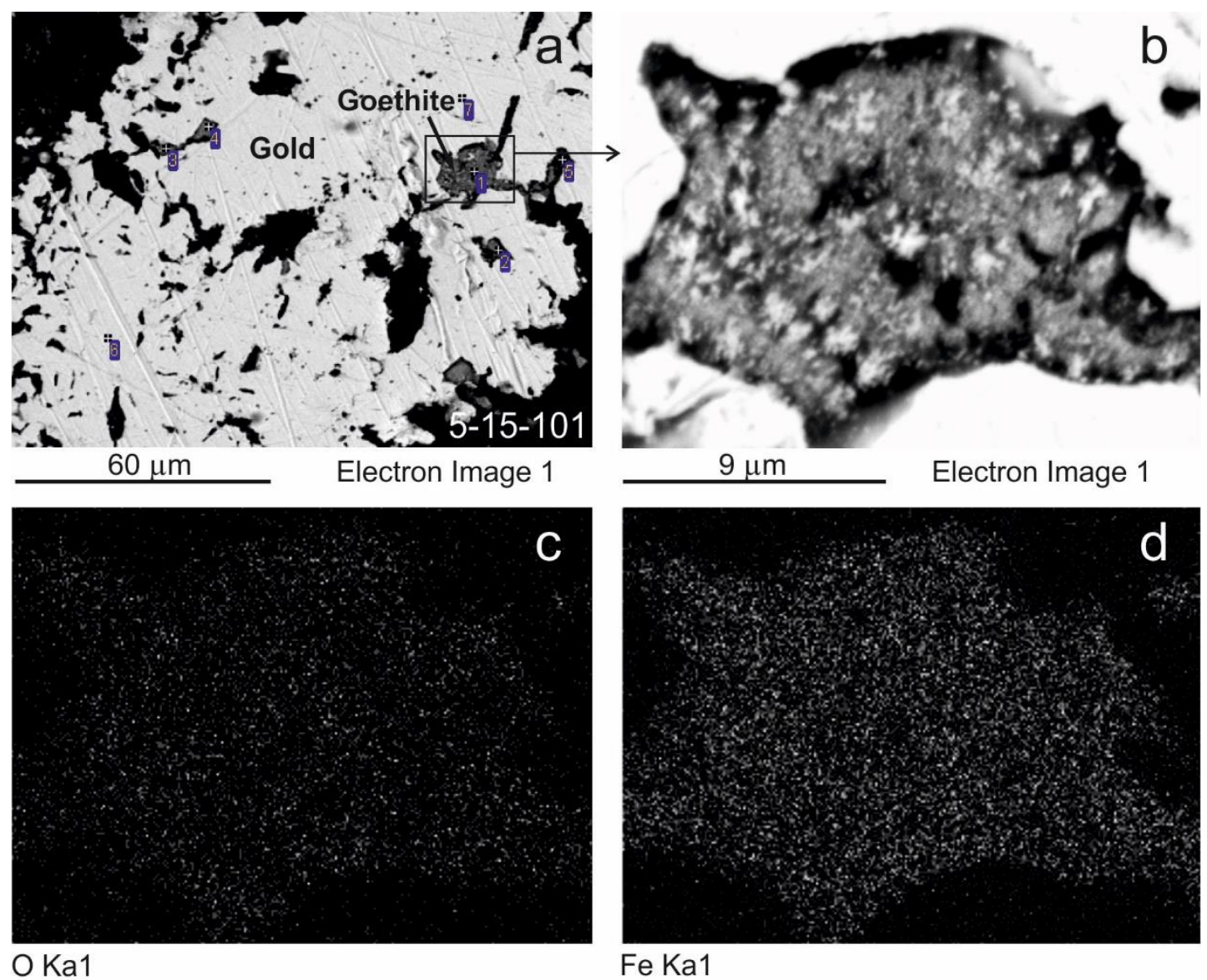

Fe Ka1
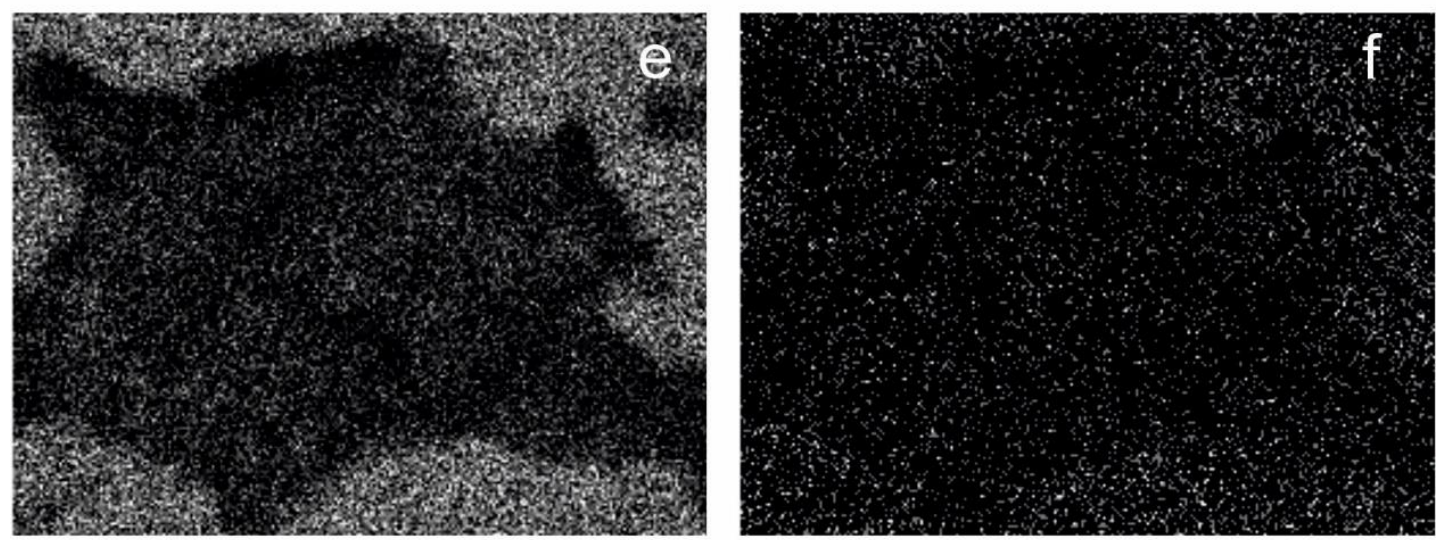

Au Ma1

Ag La1

Figure 6. Massive gold with goethite: (a) general view, (b) enlarged goethite fragment with microinclusions of gold in backscattered electrons, (c-f) in X-rays of $\mathrm{O}, \mathrm{Fe}, \mathrm{Au}$ and $\mathrm{Ag}$. 
Table 4. The composition (in wt $\%$ ) and fineness (in \%o) of the massive gold (in wt $\%$ ) shown in Figure 11.

\begin{tabular}{|c|c|c|c|c|c|c|c|c|c|c|c|}
\hline No. & Sample & $\mathrm{Fe}$ & $\mathrm{Cu}$ & Pt & Ag & $\mathrm{Au}$ & $\mathrm{Hg}$ & $\mathbf{P b}$ & Bi & Total & Fineness \\
\hline 1 & $5-15-53$ & 0.01 & - & - & 1.29 & 100.15 & 0.01 & 0.10 & 0.17 & 101.73 & 984 \\
\hline 2 & $5-15-54$ & 0.05 & - & - & 3.18 & 94.70 & 0.10 & 0.03 & - & 98.06 & 965 \\
\hline 3 & $5-15-57$ & 0.06 & 0.00 & 0.11 & 0.28 & 97.43 & 0.08 & 0.21 & 0.19 & 98.35 & 990 \\
\hline 4 & $5-15-58$ & 0.04 & - & 0.07 & 1.56 & 97.17 & 0.10 & 0.08 & 0.12 & 99.14 & 980 \\
\hline 5 & $5-15-59$ & 0.03 & - & 0.08 & 4.71 & 93.17 & - & 0.10 & 0.18 & 98.27 & 947 \\
\hline 6 & $5-15-60$ & 0.02 & - & 0.01 & 1.59 & 98.26 & 0.02 & 0.07 & 0.08 & 100.05 & 981 \\
\hline 7 & 5-15-64 & 0.01 & - & 0.01 & 1.40 & 96.88 & 0.02 & 0.07 & 0.18 & 98.57 & 982 \\
\hline 8 & $5-15-65$ & - & - & - & 0.93 & 99.64 & 0.10 & 0.10 & 0.13 & 100.89 & 987 \\
\hline 9 & $5-15-67$ & 0.01 & - & 0.04 & 1.58 & 97.67 & 0.07 & 0.02 & 0.06 & 99.45 & 981 \\
\hline 10 & 5-15-68 & - & - & - & 1.13 & 97.37 & 0.14 & 0.10 & 0.23 & 98.97 & 983 \\
\hline 11 & 5-15-69 & 0.01 & 0.00 & 0.09 & 0.83 & 96.84 & 0.14 & 0.10 & 0.18 & 98.19 & 985 \\
\hline 12 & $5-15-70$ & 0.01 & 0.02 & - & 1.48 & 99.47 & 0.08 & 0.20 & 0.22 & 101.48 & 980 \\
\hline 13 & $5-15-71$ & - & 0.01 & 0.16 & 1.11 & 99.05 & - & 0.11 & 0.21 & 100.66 & 984 \\
\hline 14 & $5-15-72$ & 0.01 & - & 0.01 & 1.02 & 100.11 & 0.08 & 0.06 & 0.12 & 101.39 & 987 \\
\hline 15 & $5-15-73$ & - & 0.01 & 0.05 & 0.73 & 99.35 & 0.03 & 0.05 & 0.15 & 100.38 & 990 \\
\hline 16 & $5-15-74$ & 0.01 & - & - & 0.60 & 100.57 & 0.01 & 0.06 & 0.12 & 101.38 & 992 \\
\hline 17 & $5-15-76$ & - & 0.00 & 0.14 & 1.33 & 96.65 & 0.05 & 0.03 & 0.10 & 98.30 & 982 \\
\hline 18 & $5-15-77$ & - & - & - & 1.46 & 97.81 & 0.13 & 0.03 & 0.06 & 99.48 & 983 \\
\hline 19 & $5-15-78$ & 0.00 & 0.01 & - & 0.71 & 99.74 & - & 0.12 & - & 100.58 & 992 \\
\hline 20 & $5-15-80$ & 0.01 & 0.01 & 0.13 & 0.92 & 97.97 & 0.11 & 0.05 & 0.19 & 99.38 & 986 \\
\hline 21 & $5-15-81$ & 0.02 & 0.02 & 0.13 & 0.90 & 97.48 & 0.10 & - & 0.14 & 98.78 & 986 \\
\hline 22 & $5-15-82$ & 0.04 & 0.02 & 0.05 & 0.91 & 98.35 & 0.03 & 0.12 & 0.17 & 99.68 & 987 \\
\hline 23 & $5-15-84$ & 0.00 & 0.02 & 0.15 & 0.88 & 98.13 & 0.01 & - & 0.19 & 99.38 & 987 \\
\hline 24 & $5-15-85$ & - & 0.01 & 0.01 & 7.46 & 90.65 & 0.04 & - & 0.03 & 98.20 & 923 \\
\hline 25 & $5-15-86$ & 0.07 & 0.02 & 0.05 & 5.13 & 92.93 & 0.26 & 0.06 & 0.03 & 98.55 & 942 \\
\hline 26 & $5-15-87$ & 0.01 & 0.01 & 0.04 & 1.00 & 98.02 & 0.19 & 0.13 & 0.09 & 99.47 & 985 \\
\hline 27 & $5-15-90$ & - & 0.01 & 0.02 & 1.45 & 96.79 & 0.02 & 0.05 & 0.19 & 98.53 & 982 \\
\hline 28 & 5-15-94 & - & 0.01 & - & 1.29 & 99.27 & 0.12 & 0.08 & 0.25 & 101.02 & 983 \\
\hline 29 & 5-15-95 & 0.12 & - & 0.05 & 8.14 & 92.21 & - & 0.05 & 0.02 & 100.60 & 917 \\
\hline 30 & 5-15-97 & 0.01 & 0.11 & 0.08 & 2.99 & 97.31 & 0.09 & 0.03 & 0.10 & 100.79 & 965 \\
\hline 31 & $5-15-98$ & - & 0.13 & 0.06 & 2.62 & 98.32 & - & 0.00 & - & 101.20 & 972 \\
\hline 32 & $5-15-100$ & - & 0.09 & 0.10 & 3.20 & 94.78 & 0.04 & 0.02 & 0.06 & 98.30 & 964 \\
\hline 33 & 5-15-101 & - & 0.08 & 0.08 & 7.27 & 90.71 & 0.11 & - & 0.05 & 98.35 & 922 \\
\hline 34 & $5-15-102$ & 0.00 & 0.03 & 0.01 & 7.41 & 90.18 & 0.01 & 0.07 & 0.04 & 97.88 & 921 \\
\hline 35 & 5-15-103 & 0.00 & 0.08 & 0.04 & 4.12 & 93.65 & 0.05 & 0.07 & 0.10 & 98.13 & 954 \\
\hline 36 & 5-15-105 & - & 0.07 & 0.07 & 7.39 & 91.03 & - & 0.02 & 0.23 & 98.84 & 921 \\
\hline 37 & 5-15-106 & - & 0.06 & 0.09 & 7.16 & 91.45 & - & - & - & 98.79 & 926 \\
\hline 38 & 5-15-107 & 0.15 & 0.01 & 0.08 & 15.95 & 81.64 & - & 0.05 & 0.01 & 97.93 & 834 \\
\hline 39 & 5-15-108 & 0.00 & 0.09 & 0.14 & 3.77 & 94.80 & - & 0.04 & 0.17 & 99.09 & 957 \\
\hline 40 & $5-15-110$ & 0.11 & 0.04 & 0.17 & 8.49 & 87.04 & 0.16 & 0.77 & 0.17 & 96.96 & 898 \\
\hline 41 & 5-15-111 & - & 0.04 & 0.06 & 7.17 & 91.49 & 0.06 & 0.04 & 0.20 & 99.08 & 923 \\
\hline 42 & $5-15-112$ & 0.00 & 0.05 & 0.01 & 6.88 & 92.73 & - & 0.02 & 0.14 & 99.82 & 929 \\
\hline 43 & $5-15-113$ & 0.01 & 0.01 & - & 7.36 & 92.62 & 0.06 & 0.06 & 0.15 & 100.28 & 924 \\
\hline 44 & $5-15-115$ & - & 0.08 & 0.05 & 3.68 & 97.45 & - & 0.01 & 0.15 & 101.42 & 961 \\
\hline 45 & $5-15-116$ & - & 0.08 & 0.03 & 3.37 & 97.24 & - & 0.04 & 0.09 & 100.86 & 964 \\
\hline 46 & 5-15-117 & 0.00 & 0.07 & 0.07 & 7.09 & 91.55 & - & - & 0.14 & 98.96 & 925 \\
\hline 47 & 5-15-118 & 0.11 & 0.01 & 0.05 & 6.47 & 93.29 & 0.06 & - & - & 100.04 & 933 \\
\hline
\end{tabular}

Note: Analyzes performed on an X-ray microanalyzer.

\subsubsection{Porous Gold}

Besides the massive gold, porous gold particles can be found. Prevalent are irregular mass, with lesser flattened forms. Both varieties are characterized by microporosity and a mustard color, indicating a wide occurrence of mustard gold in the Khokhoy gold ore field (Figure 7). The term "mustard gold" was got in by W. Lindgren [11]. Typical features of mustard gold are low reflectivity, porous or colloidal texture and rusty, reddish, orange-red and brown-yellow colors in reflected light. It is characteristic of gold-telluride and gold-antimony deposits. 

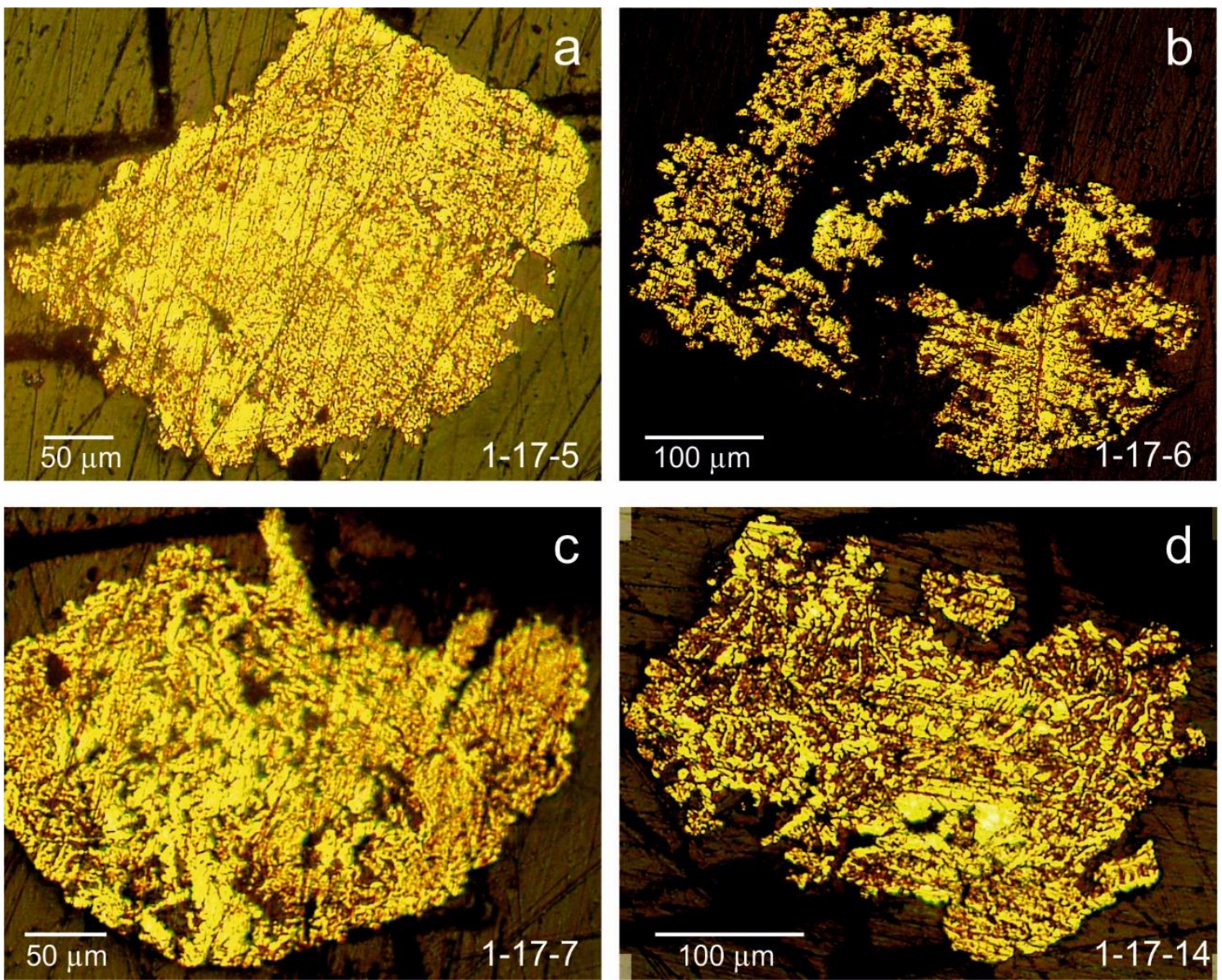

Figure 7. Native gold of the Khokhoy gold ore field, (a) microporous spongy-mustard gold, (b) framework gold with massive gold in the center, (c,d) dendritic mustard gold. Mounted sections, ore microscope, image in reflected light.

"Mustard" gold in the ore field according to the internal structure is microporous and dendritic. Porous irregular mass and flattened gold are friable aggregates of fulvous color. The porosity of gold is clearly reflected in the investigation of gold in a scanning electron microscope. The closeness of mustard and sponge gold is often observed (Figure $8 \mathrm{a}, \mathrm{b}$ ). Sometimes, massive gold grains occur with the development of hollow spongy and mustard gold closer to the center (Figure 8c). Less commonly observed is microporous mustard gold with elongated massive gold fibers in the central part (Figure $8 \mathrm{~d}$ ). The mustard gold micropores can be hollow or filled with various chemical elements $\mathrm{Fe}, \mathrm{Te}, \mathrm{Cu}, \mathrm{Mn}, \mathrm{Sb}$, and $\mathrm{Tl}$. 

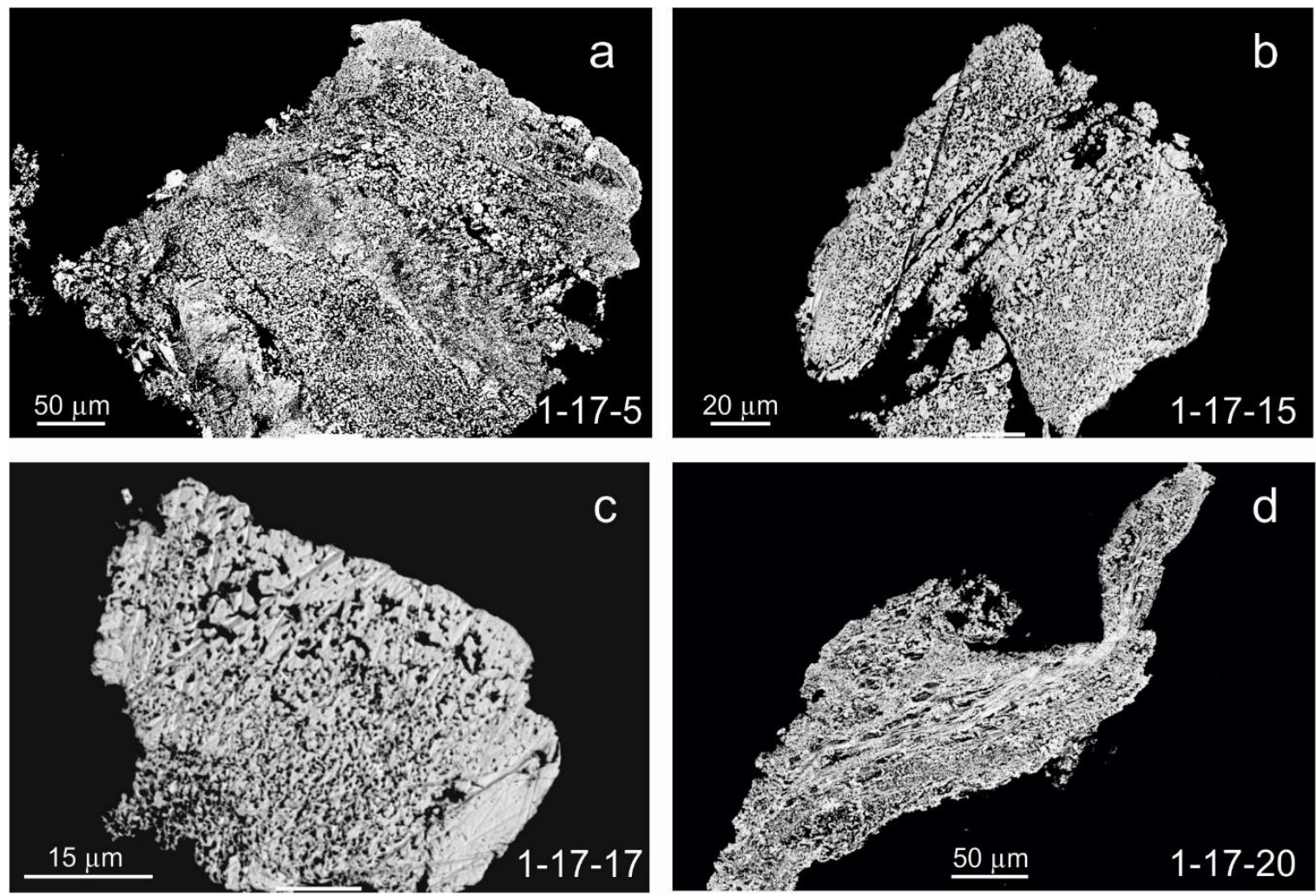

Figure 8. Mustard and sponge gold of microporous structure: $(\mathbf{a}, \mathbf{b})$ closely located mustard and sponge gold, (c) massive gold with spongy and mustard gold close the center, (d) microporous mustard gold with elongated fibers of massive gold in the center. Mounted sections, scanning electron microscope, image in the backscattering electron mode.

Mustard gold of dendritic structure is more widespread (Figure 9). Dendrites are represented in the center by dendritic-branched lumps of gold surrounded by spongy mustard gold (Figure 9a) in close association with iron hydroxides. Sometimes there are looped massive gold grains interspersed with mustard-sponge gold (Figure $9 \mathrm{~b}$ ). Varieties of mustard twiggy gold prevail, the pores of which are mostly hollow (Figure 9c,d).

According to microprobe analyses, in individual grains one can assume the presence of iron oxides (hydroxides) in the pores. Figure 10 shows the internal structure of the enlarged fragments of dendritic mustard gold. 

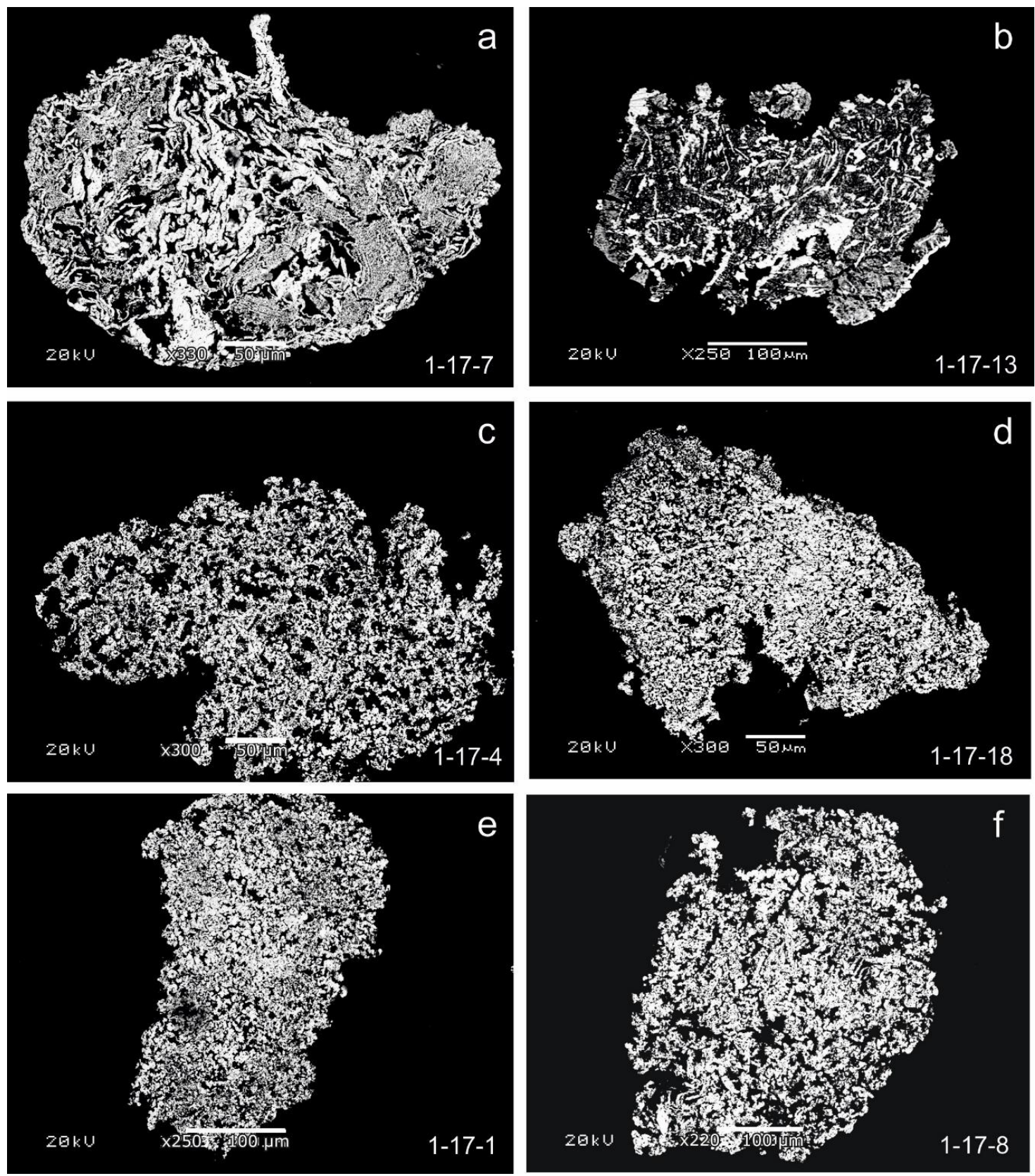

Figure 9. Mustard gold of dendritic structure, $(\mathbf{a}, \mathbf{b})$ dendritic gold in the center, meandering massive golds, mustard porous gold along the edges in close association with iron hydroxides, (c-f) varieties of twiggy gold. Mounted sections, scanning electron microscope, image in the backscattering electron mode. 

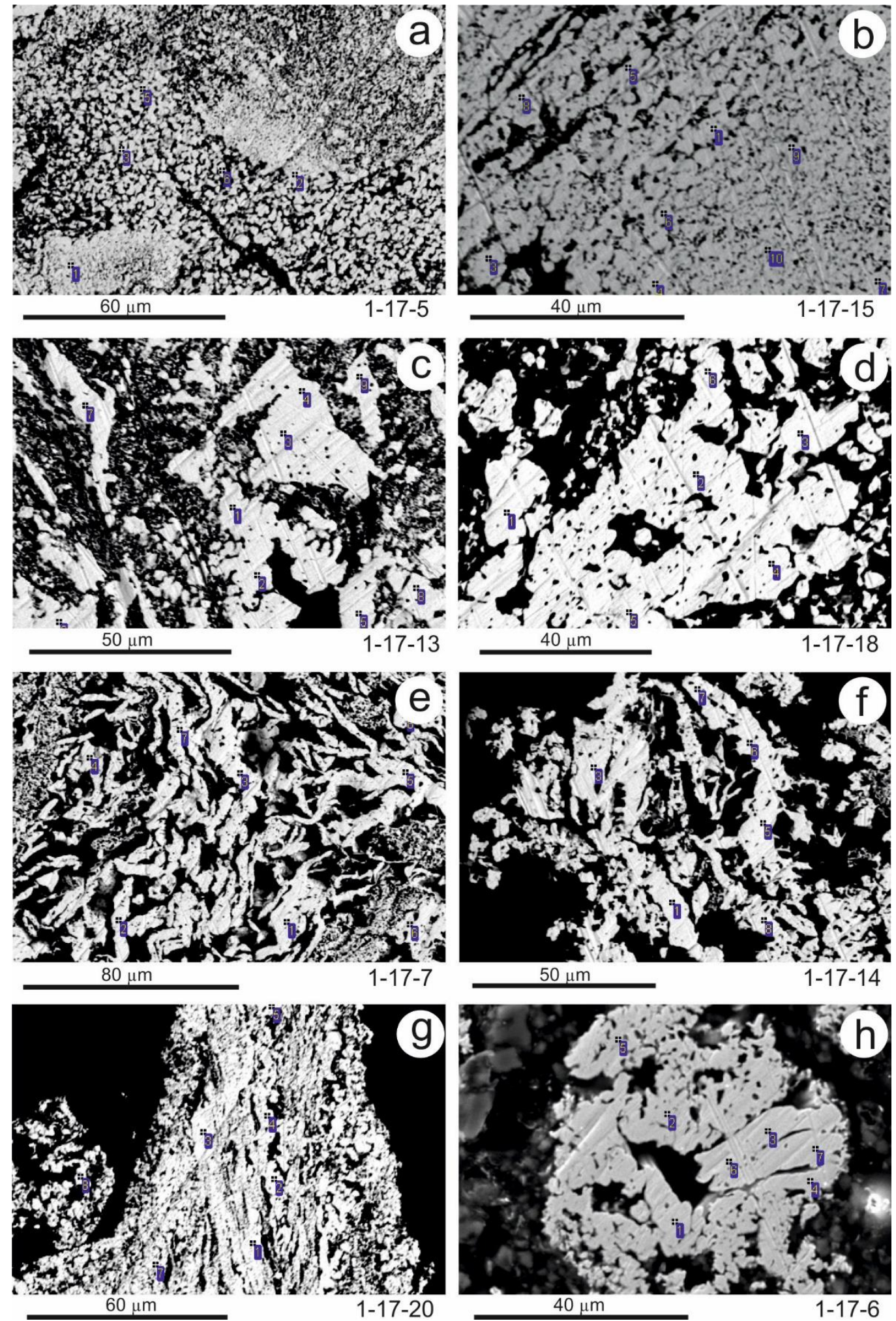

Figure 10. The internal structure of mustard-spongy $(\mathbf{a}, \mathbf{b}, \mathbf{h})$ and dendritic mustard (c-g) gold. Fragments of the gold shown in Figures 6-8. (a,b) brain structure; $(\mathbf{c}, \mathbf{d})$ massive golds are cemented with microporous mustard gold; (e,f) tortuous gold in the cement of microporous mustard-sponge gold; (g) streaky-meandering golds in the center are bordered with brain-shaped golds; (h) a lump of gold of the brain-structure. Mounted sections, scanning electron microscope, back-scattering electron mode. 
Sponge and mustard gold fineness is above $>900 \%$. Among impurities, in addition to silver, $\mathrm{Hg}$ (up to $5.48 \mathrm{wt} \%$ ) and $\mathrm{Bi}$ (up to $0.42 \mathrm{wt} \%$ ) are constantly present, the remaining elements $\mathrm{Fe}, \mathrm{Zn}, \mathrm{Pb}, \mathrm{Cu}$, and Pt are found sporadically (Table 2, Nos. 11-56, Table 5).

Table 5. The composition (in $\mathrm{wt} \%$ ) and fineness (in \%o) of the porous gold shown in Figure 11.

\begin{tabular}{|c|c|c|c|c|c|c|c|c|c|c|c|c|}
\hline No. & Sample & Sp. & $\mathrm{Fe}$ & $\mathrm{Cu}$ & $\mathrm{Zn}$ & $\mathrm{Ag}$ & $\mathrm{Au}$ & $\mathrm{Hg}$ & $\mathrm{Pb}$ & $\mathrm{Bi}$ & Total & Fineness \\
\hline 1 & $1-17-1$ & 1 & - & 0.02 & - & 4.79 & 94.29 & 0.15 & - & 0.15 & 99.40 & 949 \\
\hline 2 & $1-17-1$ & 2 & 0.06 & - & 0.01 & 1.01 & 96.85 & 0.07 & 0.08 & 0.10 & 98.17 & 986 \\
\hline 3 & $1-17-2$ & 1 & 0.05 & 0.02 & - & 8.86 & 89.44 & - & 0.08 & 0.05 & 98.50 & 908 \\
\hline 4 & $1-17-6$ & 1 & 0.05 & 0.02 & - & 0.852 & 97.14 & 0.02 & 0.01 & 0.16 & 98.25 & 989 \\
\hline 5 & $1-17-6$ & 2 & 0.03 & 0.02 & 0.02 & 0.717 & 99.28 & 0.17 & - & 0.22 & 100.46 & 988 \\
\hline 6 & $1-17-6$ & 3 & 0.03 & 0.01 & - & 2.52 & 94.86 & 0.22 & 0.01 & 0.08 & 97.73 & 971 \\
\hline 7 & $1-17-7$ & 1 & - & 0.04 & - & 0.752 & 98.68 & 0.09 & 0.03 & 0.15 & 99.74 & 989 \\
\hline 8 & $1-17-7$ & 2 & - & 0.02 & - & 0.092 & 99.74 & 0.25 & 0.08 & 0.16 & 100.34 & 994 \\
\hline 9 & $1-17-7$ & 3 & 0.05 & 0.01 & 0.01 & 0.194 & 99.6 & 0.26 & 0.04 & 0.26 & 100.42 & 992 \\
\hline 10 & $1-17-7$ & 4 & 0.06 & 0.02 & - & 0.627 & 97.98 & 0.14 & - & 0.12 & 98.945 & 990 \\
\hline 11 & $1-17-8$ & 1 & - & - & - & 5.17 & 95.24 & - & 0.02 & 0.10 & 100.54 & 947 \\
\hline 12 & $1-17-8$ & 2 & - & - & - & 5.67 & 95.96 & 0.07 & 0.04 & 0.09 & 101.84 & 942 \\
\hline 13 & $1-17-8$ & 3 & - & 0.02 & - & 6.07 & 94.35 & 0.27 & - & 0.07 & 100.77 & 936 \\
\hline 14 & $1-17-8$ & 4 & - & 0.02 & 0.01 & 5.09 & 91.64 & 0.18 & 0.04 & 0.12 & 97.1 & 944 \\
\hline 15 & $1-17-8$ & 5 & - & - & - & 4.89 & 95.79 & 0.29 & 0.02 & 0.14 & 101.13 & 947 \\
\hline 16 & $1-17-9$ & 1 & - & - & - & 6.04 & 92.1 & 0.27 & - & 0.01 & 98.42 & 936 \\
\hline 17 & $1-17-9$ & 2 & 0.01 & 0.01 & - & 3.28 & 96.79 & 0.17 & - & 0.14 & 100.40 & 964 \\
\hline 18 & $1-17-10$ & 1 & - & 0.01 & - & 1.07 & 98.75 & 0.02 & 0.11 & 0.16 & 100.11 & 986 \\
\hline 19 & $1-17-10$ & 2 & - & 0.01 & - & 1.16 & 99.94 & 0.32 & 0.09 & 0.13 & 101.66 & 983 \\
\hline 20 & $1-17-10$ & 3 & - & - & - & 1.19 & 99.93 & 0.10 & - & 0.28 & 101.50 & 985 \\
\hline 21 & $1-17-11$ & 1 & - & - & - & 1.42 & 98.56 & 0.24 & 0.02 & 0.25 & 100.49 & 981 \\
\hline 22 & $1-17-11$ & 2 & - & 0.01 & 0.012 & 3.99 & 96.46 & 0.19 & - & 0.17 & 100.83 & 957 \\
\hline 23 & $1-17-11$ & 3 & - & 0.01 & - & 3.95 & 96.14 & 0.35 & 0.03 & 0.18 & 100.66 & 955 \\
\hline 24 & $1-17-12$ & 1 & - & - & - & 2.56 & 97.78 & 0.20 & 0.07 & 0.02 & 100.63 & 972 \\
\hline 25 & $1-17-13$ & 1 & - & 0.03 & - & - & 99.62 & - & - & 0.24 & 99.89 & 997 \\
\hline 26 & $1-17-14$ & 1 & 0.07 & - & - & 0.79 & 98.82 & 0.1 & 0.05 & 0.22 & 100.04 & 988 \\
\hline 27 & $1-17-15$ & 1 & 0.03 & - & 0.03 & 0.30 & 96.62 & 0.30 & 0.10 & 0.24 & 97.62 & 990 \\
\hline 28 & $1-17-17$ & 1 & - & - & 0.02 & 0.29 & 99.72 & 0.27 & 0.01 & 0.24 & 100.56 & 992 \\
\hline 29 & $1-17-18$ & 1 & - & - & - & 3.87 & 90.87 & 4.63 & - & 0.18 & 99.55 & 913 \\
\hline 30 & $1-17-19$ & 1 & 0.04 & - & - & 6.78 & 91.49 & 2.97 & - & 0.066 & 101.35 & 903 \\
\hline 31 & $1-17-21$ & 1 & - & - & - & 1.88 & 97.46 & 0.22 & 0.03 & 0.42 & 100.01 & 975 \\
\hline 32 & $1-17-21$ & 2 & 0.02 & 0.02 & 0.01 & 4.00 & 93.29 & 0.04 & - & 0.18 & 97.56 & 956 \\
\hline
\end{tabular}

Note: Analyzes performed on an X-ray microanalyzer.

All gold varieties are chemically homogeneous and characterized by high fineness of the gold particles without any rims or margins. The distribution patterns of fineness in both types of native gold massive and porous, do not differ essentially. The high-fineness gold ( $>950 \%$ ) makes up $64 \%$, the amount of particles of lower fineness (800-950\%o) comes to only 36\% of the total. Along with Ag, the porous native gold invariably contains mercury (up to $5.78 \mathrm{wt} \%$ ) and bismuth, and, less frequently, lead, copper, and iron.

Cumulative diagrams clearly show high concentrations of $\mathrm{Pb}$ and $\mathrm{Cu}$ in the massive gold, and of $\mathrm{Hg}$ in the porous one (Figure 11). The amount of Fe and Bi impurities in both types of supergene gold is similar. 

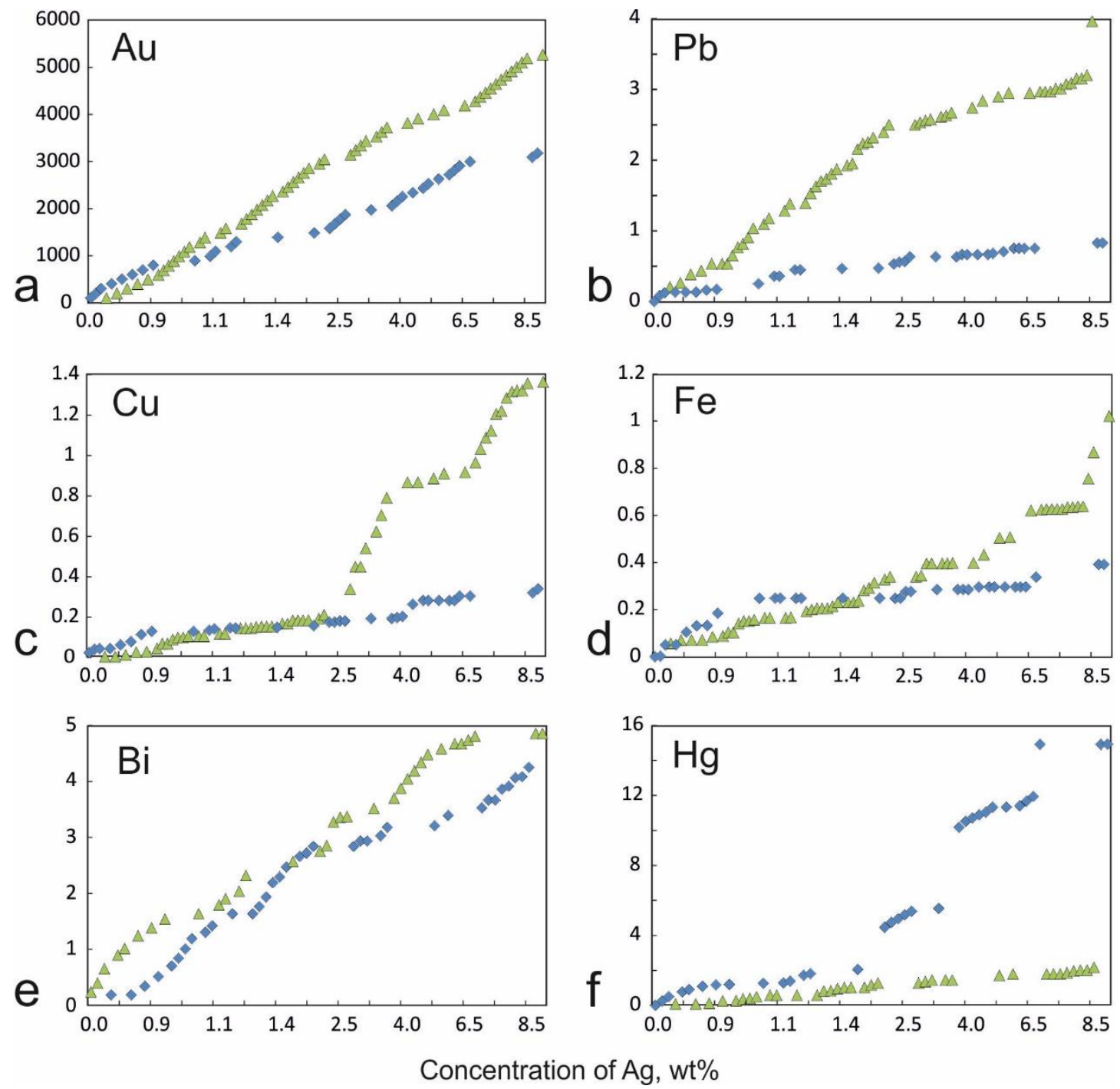

Figure 11. Cumulative plots of trace element ((a) $\mathrm{Au},(\mathbf{b}) \mathrm{Pb},(\mathbf{c}) \mathrm{Cu},(\mathbf{d}) \mathrm{Fe},(\mathbf{e}) \mathrm{Bi},(\mathbf{f}) \mathrm{Hg})$ vs. silver concentrations in massive (green triangle) and porouse (blue rombus) gold of the Khokhoy gold ore field.

\section{Discussion}

Two genetic types of supergene gold, massive and porous, are recognized there.

The first type is represented by gold crystals and irregular mass, with the fineness ranging from 835 to $1000 \%$ o. They are closely associated with goethite, siderite, unnamed $\mathrm{Fe}, \mathrm{Te}$, and $\mathrm{Tl}$ carbonates, $\mathrm{Tl}$ tellurites/tellurates and antimonates, as well as avicennite with a Te impurity.

The second type is represented by mustard gold with different internal structure: microporous and dendritic.

Evidentially, mustard gold is characteristic of gold-telluride [12-15] and gold-antimony [16-18] deposits, weathering crust [19-21] and placers [22,23]. Recently, mustard gold, sometimes with an admixture of $\mathrm{Tl}$ (up to $6.34 \mathrm{wt} \%$ ), was found in the Oleninsky Au-Ag deposit (Kola Peninsula, Russia) [24]. In all the cases, mustard gold was developed in the oxidation zone as a result of the decomposition of tellurides, antimonides, sulfides, bismuthides $\mathrm{Au}(\mathrm{Ag})$ and low-fineness gold [14]. In the Kuranakh deposit, mustard gold is the product of the decomposition of tellurates [25]. Researchers consider the occurrence of mustard gold a result of hypogenous as well as supergenous processes. The proponents of its hypogenic origin believe that mustard gold, Au tellurates/antimonates, and 
complex gold oxides were formed from hydrothermal low-temperature solutions with a high oxidation potential [16]. Supergene processes explain the origin of the secondary mustard gold of high fineness of placers in the North-East of Russia [23].

The formation of mustard gold at the Dongping mines (Hebei province, China) has been related to decomposition of calaverite by selective leaching of tellurium while leaving the gold alloy in the cavity formed by the alteration reaction [12,13]. This type of pseudomorphic alteration was also documented by Palache et al. [26]. The occurrence of microporous gold has also been observed under cold climatic conditions, such as at the Aginskoe low-sulfidation ephithermal deposit in Central Kamchatka, Russia [27]. In this deposit, calaverite is the main Au telluride mineral and it has been partially replaced by porous gold. By comparing the textures of microporous gold from this natural occurrence with those obtained experimentally via the dealloying of gold-(silver) tellurides [28-30], Okrugin et al. [31] confirmed that natural microporous gold can form via the replacement of telluride minerals and assessed the role that hydrothermal fluids may play in the formation of microporous gold. The formation of the secondary high-fineness mustard gold from placers in northeast Russia is interpreted to be due to supergene processes. Although no antimonides, nor tellurides, or gold bismuthides were found in the ores of the Khokhoy gold ore field, their presence is assumed by the minerals associated with supergenic gold and the microchemical composition. They are represented by Fe, Mn and Tl tellurates and carbonates, as well as avicennite, Tl antimonates, goethite, limonite, hydrogoethite, and siderite of which the breakdown contributes to the formation of microporous gold particles of spungy habit.

So, what is the formation mechanism of dendrites? Interesting data on the formation of native gold dendrites in epithermal ores are presented by Saunders [32-34]. Author showed a scanning electron microscope (SEM) image of electrum dendrites that appeared to have formed from aggregation of smaller colloid-sized particles. Metallic nanoparticles appear to form from supersaturated solutions and can form dendrites by the self-assembly and aggregation of the nanoparticles. These dendrites are typically an intermediary stage to more traditional crystal formation as the infilling of branches of the dendrites occurs. In epithermal ores, the dendrites of electrum appear to be preserved due to the infilling of other nanoparticles between the branches, such as silica nanoparticles. The 'fractal' electrum dendrites have been observed in many the Tertiary bonanza epithermal ores in northern Nevada, and have been interpreted to be evidence of nanoparticle nucleation and aggregation in ore formation. More recently, similar textures and genetic interpretations have been made from ores from the southeastern USA [33] and Bulgaria [35]. Finally, disseminated electrum nanoparticles have recently been discovered in the epithermal Round Mountain (Nevada) deposit and have been proposed to be precursors for coarser electrum crystals there [36,37].

We believe that this is a more suitable mechanism of formation for the supergene gold of the Khokhoy gold ore field. The colloidal gold transfer is thought to be possible not only in hydrothermal conditions but in supergene zones too [38]. In the oxidation zone of low-sulfidation ores, to which group the Khokhoy ores belong, the role of gold colloids could be significant. Nanoparticles or colloids of gold with nanoparticles of silica (opal, chalcedony) could form dendrites by the self-assembly and aggregation of the nanoparticles. These dendrites were an intermediary stage to the formation of massive gold particles and crystals by the infilling of the dendrite branches. The massive gold type occurs in the most hypsometrically high levels of karst formations of the Khokhoy gold ore field.

The style of mineralization is among the main factors defining the microchemical composition of native gold, particularly the placer gold [39]. In the Khokhoy gold ore field, the main ore minerals associated with primary gold are pyrite, hematite, galena, and chalcosine. Supergene gold occurs in paragenesis with goethite, siderite, oxides, carbonates, tellurates, and antimonates of thallium. The set of trace elements $(\mathrm{Cu}, \mathrm{Pb}, \mathrm{Fe})$, both in the massive and porous gold, corresponds to main elements of the associated minerals. The constant presence of $\mathrm{Bi}$ in both gold types suggests an intrusive source for them. The preferential accumulation of $\mathrm{Hg}$ in porous gold may be explained by its friable texture. 
The lack of gold placers in the Khokhoy gold ore field may be due to that microaggregates of mustard gold are instable in supergene process because of friable texture, thus having a weak potential for placer formation.

The fact that native gold is unvisible and fine-grained in primary ores and visible in the karst cavities indicates that it grew larger in size in the oxidation zone of karst formations.

\section{Conclusions}

Relationships of massive gold with unnamed tellurates and carbonates of thallium and with avicennite $\left(\mathrm{Te}_{2} \mathrm{O}_{3}\right)$ are first described for the Khokhoy gold ore field. Along with massive gold closely associated with $\mathrm{Tl}$ minerals, there are abundant porous particles, the so called spungy and mustard gold.

The research has shown that the Khokhoy gold ore field, according to its mineralogical and geochemical features, should be classified as a deposit of $\mathrm{Au}-\mathrm{Te}-\mathrm{Sb}-\mathrm{Tl}$ mineral composition localized in karst cavities. A typical gold ore deposit in karst cavities is the Kuranakh deposit of Central Aldan district of South Yakutia [6], with which our data is being compared. It should be noted that in the Kuranakh deposit one of the typomorphic geochemical elements is thallium, but its mineral form has not been identified. In the Khokhoy ore field, a diverse spectrum of thallium minerals is attested. This fact makes closer to Carlin-type gold deposits in the West of the USA [1-5], Alshar epithermal Au-As-Hg-Tl deposit in Macedonia [40,41], Vorontsovskoe deposit in the Urals, Russia [7-9,42] etc. The main difference between the Khokhoy gold ore field and these deposits at this stage of research is the absence of As and Hg minerals - realgar, auripigment, cinnabar. In addition to thallium minerals, $\mathrm{Sb}$ and Te minerals (weissbergite, antimonite, berthierite, and unnamed antimonates and tellurates of thallium) are widespread in the ores.

Supergene gold in karst cavities has specific features: (1) morphology-massive (gold crystals and cloddy particles of monolithic structure) and porous (microporous and dendritic); (2) associated minerals-goethite, limonite, avicennite, hydrogoethite, and siderite, Fe, Mn and Tl tellurates and carbonates, Tl antimonates; (3) persistently high fineness and chemically homogeneous; (4) microchemical elements $\mathrm{Hg}, \mathrm{Bi}, \mathrm{Fe}$. The obtained characteristics made it possible to prove the existence of two genetic types of supergene gold. Mustard microporous gold is the result of the decomposition of the associated goethite minerals, Tl oxides, tellurates, carbonates and antimonates $\mathrm{Fe}, \mathrm{Mn}$ and $\mathrm{Tl}$ containing microinclusions of gold. Massive gold and dendrites are newly formed. The decomposition, remobilization, and reprecipitation of residual gold nanoparticles and their aggregation led to the formation of dendrites, and with further crystal growth and filling of pores, to gold of massive morphology.

The supergene gold of the Khokhoy gold ore field is comparable in its typomorphic characteristics to that of the Kuranakh deposit, as well as with to that of Carlin-type gold deposits in the West of the USA, to that of the Alshar epithermal deposit in Macedonia, to that of the Vorontsovskoe deposit in the Urals, Russia, and also have similarities with those of sponge and mustard gold of gold-telluride and gold-antimony deposits, weathering crusts and placers.

However, at the same time, the gold has certain uniqueness, namely the paragenesis with thallium minerals. To date, no such relationship has been cited in the literature.

Author Contributions: The idea of the research belongs to G.S.A., who has then designed it, analyzed the results and wrote the paper. L.A.K. was responsible for the fieldwork in the auriferous zone; she also supported the research with valuable ideas. V.N.K. was also responsible for the fieldwork in the auriferous zone; moreover she made the mineralgraphic description and took the photographs of the polished sections. All authors have read and agreed to the published version of the manuscript.

Funding: The research was carried out under the governmental project of Diamond and Precious Metal Geology Institute, SB RAS, which was financially supported by the Ministry of science and higher education of the Russian Federation, the project number is 0381-2019-0004 and Russian Foundation for Basic Reseach, the project number is $18-45-140045$. 
Acknowledgments: We would like to thank N. Khristoforova, S. Popova and S. Karpova for their help with an enormous part of this research, analyzing the samples with scanning electronic microscope and performing X-ray spectral analysis (Diamond and Precious Metal Geology Institute, SB RAS). We are also very grateful to E. Sokolov for the organization of the fieldwork at the auriferous field (JSC 'Yakutskgeologiya'). Authors thanks anonymous reviewers for their comments and constructive suggestions.

Conflicts of Interest: We declare no conflict of interest.

\section{References}

1. Hofstra, A.H.; Cline, J.S. Characteristics and models for Carlin-type gold deposits. Econ. Geol. 2000, 13, 163-220.

2. Cline, J.S.; Hofstra, A.H.; Muntean, J.L.; Tosdal, R.M.; Kenneth, K.A. Carlin-Type Gold Deposits in Nevada: Critical Geologic Characteristics and Viable Models. Econ. Geol. 2005, 100, 451-484.

3. Volkov, A.V.; Sidorov, A.A. The geological-genetic model of Carlin type gold deposits. Litosphere 2016, 6, 145-165. (In Russian)

4. Zhong, H.R.; Chao, S.W.; Wu, B.X.; Zhi, T.G.; Hofstra, A.H. Geology and geochemistry of Carlin-type gold deposits in China. Miner. Depos. 2002, 37, 378-392. [CrossRef]

5. Xia, Y.; Su, W.; Zhang, Z.; Liu, J. Geochemistry and Metallogenic Model of Carlin-Type Gold Deposits in Southwest Guizhou Province, China. In Geochemistry Earth's System Processes; INTECH: London, UK, 2012.

6. Rodionov, S.M.; Fredericksen, R.S.; Berdnikov, N.V.; Yakubchuk, A.S. The Kuranakh epithermal gold deposit (Aldan Shield, East Russia). Ore Geol. Rev. 2014, 59, 55-65. [CrossRef]

7. Sazonov, V.N.; Murzin, V.V.; Grigor'ev, N.A. Vorontsovsk gold deposit: An example of carlin-type mineralization in the Urals, Russia. Geol. Ore Depos. 1998, 40, 139-151.

8. Murzin, V.V.; Naumov, E.A.; Azovskova, O.B.; Varlamov, D.A.; Rovnushkin, M.Y.; Pirajno, F. The Vorontsovskoe $\mathrm{Au}-\mathrm{Hg}-\mathrm{As}$ ore deposit (Northern Urals, Russia): Geological setting, ore mineralogy, geochemistry, geochronology and genetic model. Ore Geol. Rev. 2017, 85, 271-298. [CrossRef]

9. Vikentyev, I.V.; Tyukova, E.E.; Vikent'eva, O.V.; Chugaev, A.V.; Dubinina, E.O.; Prokofiev, V.Y.; Murzin, V.V. Vorontsovka Carlin-style gold deposit in the North Urals: Mineralogy, fluid inclusion and isotope data for genetic model. Chem. Geol. 2019, 508, 144-166. [CrossRef]

10. Anisimova, G.S.; Kondratieva, L.A.; Sokolov, E.P.; Kardashevskaya, V.N. Gold mineralization of the Lebedinsky and Kuranakh types in the Verkhneamginsky district (South Yakutia). Otech. Geol. 2018, 5, 3-13. (In Russian)

11. Lindgren, W. Mineral Deposits; McGraw-Hill Book Company, Inc.: New York, NY, USA; London, UK, 1933; p. 930.

12. Petersen, S.B.; Makovicky, E.; Li, J.L.; Rose-Hansen, J. Mustard gold from the Dongping Au-Te deposit, Hebei province, People's Republic of China. Neues Jahrb. Mineral. Mh. 1999, 8, 337-357.

13. Li, J.; Makovicky, E. New studies on mustard gold from the Dongping Mines, Hebei Province, China: The tellurian, plumbian, manganoan and mixed varieties. Neues Jahrb. Mineral. Abh. 2001, 176, $269-297$.

14. Tolstykh, N.D.; Palyanova, G.A.; Bobrova, O.V.; Sidorov, E.G. Mustard Gold of the Gaching Ore Deposit (Maletoyvayam Ore Field, Kamchatka, Russia). Minerals 2019, 9, 489-506. [CrossRef]

15. Tolstykh, N.; Vymazalova, A.; Tuhy, M.; Shapovalova, M. Conditions of formation of Au-Se-Te mineralization in the Gaching ore occurrence (Maletoyvayam ore field), Kamchatka, Russia. Mineral. Mag. 2018, 82, 649-674. [CrossRef]

16. Gamyanin, G.N.; Nekrasov, I.; Zhdanov, J.J.; Leskova, I.V. Auroantimonate-A new natural compound of gold. Dokl. Akad. Nauk SSSR 1988, 301,947-950. (In Russian)

17. Amuzinsky, V.A.; Anisimova, G.S.; Zhdanov, Y.Y. Sarylakhskoe and Sentachanskoe Gold Antimony Deposits; Nauka/Interperiodika: Moscow, Russia, 2001; 218p. (In Russian)

18. Zacharias, J.; Nemec, M. Gold to aurostibite transformation and formation of Au-Ag-Sb phases: The Krásná Hora deposit, Czech Republic. Mineral. Mag. 2017, 81, 987-999. [CrossRef]

19. Kalinin, Y.A.; Kovalev, K.R.; Naumov, E.A.; Kirillov, M.V. Gold in the weathering crust the Suzdal' deposit (Kazakhstan). Russ. Geol. Geogphys. 2009, 50, 174-187. [CrossRef]

20. Kalinin, Y.A.; Palyanova, G.A.; Bortnikov, N.S.; Naumov, E.A.; Kovalev, K.R. Aggregation and Differentiation of Gold and Silver during the Formation of the Gold-Bearing Weathering Crusts (on the Example of Kazakhstan Deposits). Dokl. Earth Sci. 2018, 482, 1193-1198. [CrossRef] 
21. Kalinin, Y.A.; Palyanova, G.A.; Naumov, E.A.; Kovalev, K.R.; Pirajno, F. Supergene remobilization of Au in Au-bearing regolith related to orogenic deposits: A case study from Kazakhstan. Ore Geol. Rev. 2019, 109, 358-369. [CrossRef]

22. Reith, F.; Stewart, L.; Wakelin, S.A. Supergene Au transformation: Secondary and nano-particulate Au from southern New Zealand. Chem. Geol. 2012, 320-321, 32-45. [CrossRef]

23. Litvinenko, I.S.; Shilina, L.A. Hypergene gold neomineralization in placer deposits of Nizhne-Myakitsky ore-placer field, North-East Russia. J. Ores Met. 2017, 1, 75-90. (In Russian)

24. Kalinin, A.A.; Savchenko, Y.E.; Selivanova, E.A. Mustard Gold in the Oleninskoe Gold Deposit, Kolmozero-Voronya Greenstone Belt, Kola Peninsula, Russia. Minerals 2019, 9, 786-800. [CrossRef]

25. Yablokova, S. About a new morphological variety of gold and its origin. Dokl. Akad. Nauk SSSR 1972, 205, 936-939. (In Russian)

26. Palache, C.; Berman, H.; Frondel, C. Dana's System of Mineralogy I; Wiley: New York, NY, USA, 1944.

27. Andreeva, E.D.; Matsueda, H.; Okrugin, V.M.; Takahashi, R.; Ono, S. Au-Ag-Te mineralization of the low-sulfidation epithermal Aginskoe deposit, Central Kamchatka, Russia. Resour. Geol. 2013, 63, 337-349. [CrossRef]

28. Xu, W.; Zhao, J.; Brugger, J.; Chen, G.; Pring, A. Mechanism of mineral transformations in krennerite, Au3AgTe8, under hydrothermal conditions. Am. Miner. 2013, 98, 2086-2095. [CrossRef]

29. Zhao, J.; Xia, F.; Pring, A.; Brugger, J.; Grundler, P.V.; Chen, G. A novel pre-treatment of calaverite by hydrothermal mineral replacement reactions. Miner. Eng. 2010, 23, 451-453. [CrossRef]

30. Zhao, J.; Brugger, J.; Xia, F.; Ngothai, Y.; Chen, G.; Pring, A. Dissolution-reprecipitation vs. solid-state diffusion: Mechanism of mineral transformations in sylvanite, $(\mathrm{AuAg})_{2} \mathrm{Te}_{4}$, under hydrothermal conditions. Am. Miner. 2013, 98, 19-32. [CrossRef]

31. Okrugin, V.M.; Andreeva, E.; Etschmann, B.; Pring, A.; Li, K.; Zhao, J.; Griffiths, G.; Lumpkin, G.R.; Triani, G.; Brugger, J. Microporous gold: Comparison of textures from Nature and experiments. Am. Miner. 2014, 99, 1171-1174. [CrossRef]

32. Saunders, J.A.; Schoenly, P.A. Boiling, colloid nucleation and aggregation, and the genesis of bonanza gold mineralization at the Sleeper Deposit, Nevada. Miner. Depos. 1995, 30, 199-211. [CrossRef]

33. Saunders, J.A. Textural evidence of episodic introduction of metallic nanoparticles into bonanza epithermal ores. Minerals 2012, 2, 228-243. [CrossRef]

34. Saunders, J.A.; Burke, M. Formation and aggregation of gold (electrum) nanoparticles in epithermal ores. Minerals 2017, 7, 163-173. [CrossRef]

35. Marinova, I.; Ganev, V.; Titorenkova, R. Colloidal origin of colloform-banded textures in the Paleogene low-sulfidation Khan Krum gold deposit, SE Bulgaria. Miner. Depos. 2014, 49, 49-74. [CrossRef]

36. Burke, M. An Electron Microscopy Investigation of Gold and Associated Minerals from Round Mountain, Nevada. Master's Thesis, Miami University, Oxford, OH, USA, 2017.

37. Burke, M.; Rakovan, J.; Krekeler, M.P.S. A study by electron microscopy of gold and associated minerals from Round Mountain, Nevada. Ore Geol. Rev. 2017, 91, 708-717. [CrossRef]

38. Petrovskaya, N.V. Native Gold; Nauka: Moscow, Russian, 1973; 347p. (In Russian)

39. Chapman, R.J.; Mortensen, J.K.; LeBarge, W.P. Styles of lode gold mineralization contributing to the placers of the Indian River and Black Hills Creek, Yukon Territory, Canada as deduced from microchemical characterization of placer gold grains. Miner. Depos. 2011, 46, 881-903. [CrossRef]

40. Volkov, A.V.; Serafimovski, T.; Kochneva, N.T.; Tomson, I.N.; Tasev, G. The Alshar epithermal Au-As-Sb-Tl deposit, southern Macedonia. Geol. Ore Depos. 2006, 48, 175-192. [CrossRef]

41. Palinkaš, S.S.; Hofstra, A.H.; Percival, T.J.; Šoštari'c, S.B.; Palinkaš, L.; Bermanec, V.; Pecskay, Z.; Boev, B. Comparison of the Allchar Au-As-Sb-Tl Deposit, Republic of Macedonia, with Carlin-Type Gold Deposits. Rev. Econ. Geol. 2018, 20, 335-363.

42. Kasatkin, A.V.; Makovicky, E.; Plášil, J.; Škoda, R.; Agakhanov, A.A.; Karpenko, V.Y.; Nestola, F. Tsygankoite, $\mathrm{Mn}_{8} \mathrm{Tl}_{8} \mathrm{Hg}_{2}\left(\mathrm{Sb}_{21} \mathrm{~Pb}_{2} \mathrm{Tl}_{\Sigma 24} \mathrm{~S}_{48}\right.$, a New Sulfosalt from the Vorontsovskoe Gold Deposit, Northern Urals, Russia. Minerals 2018, 8, 218. [CrossRef]

(C) 2020 by the authors. Licensee MDPI, Basel, Switzerland. This article is an open access article distributed under the terms and conditions of the Creative Commons Attribution (CC BY) license (http://creativecommons.org/licenses/by/4.0/). 